\title{
Possible Worlds Semantics for Partial Meet Multiple Contraction
}

\author{
Maurício D. L. Reis • Eduardo Fermé
}

Received: 2 January 2010 / Accepted: 1 May 2011 / Published online: 31 May 2011

(c) Springer Science+Business Media B.V. 2011

\begin{abstract}
In the logic of theory change, the standard model is AGM, proposed by Alchourrón et al. (J Symb Log 50:510-530, 1985). This paper focuses on the extension of AGM that accounts for contractions of a theory by a set of sentences instead of only by a single sentence. Hansson (Theoria 55:114-132, 1989), Fuhrmann and Hansson (J Logic Lang Inf 3:39-74, 1994) generalized Partial Meet Contraction to the case of contractions by (possibly non-singleton) sets of sentences. In this paper we present the possible worlds semantics for partial meet multiple contractions.
\end{abstract}

Keywords Belief change - Theory contraction • Multiple contraction • Possible worlds semantics

\section{Introduction}

One of the main concerns of the logic of theory change consists in modeling how information is removed from the set of beliefs of an agent; in other words, one of its fundamental purposes is to find appropriate ways of defining contraction operations. Roughly speaking, given a set of beliefs, such a function

\footnotetext{
Maurício D. L. Reis was supported by Fundação para a Ciência e a Tecnologia (FCT) through the grant SFRH/BD/30175/2006 financed by national funds from Ministério da Ciência, Tecnologia e Ensino Superior (MCTES) from Portugal.

M. D. L. Reis ( $\varangle)$

Centro de Ciências Exactas e da Engenharia and Madeira Interactive Technologies Institute, Universidade da Madeira, Campus Universitário da Penteada, 9020-105 Funchal, Portugal e-mail: m_reis@uma.pt

E. Fermé

Madeira Interactive Technologies Institute, Universidade da Madeira, Funchal, Portugal e-mail: ferme@uma.pt
} 
receives a sentence or a set of sentences that is intended to be removed from it and returns a new set of beliefs that no longer contains the given sentence(s).

All earlier investigations of this processes [1,9-12] dealt essentially with the removal of a specific sentence from a belief set - a set of sentences closed under logical consequence. The first and most widely known constructive model of such an operation is the partial meet contraction [1]. The possible worlds semantics for this operation was introduced in [12].

However, in a subsequent stage of the development of the theory of belief contraction, several researchers (e.g. $[6-8,13,16])$ pointed out the need for defining operations that could account for changes consisting of the removal of sets with more than one element. In this respect, Fuhrmann and Hansson [8] remarked that contracting a belief set $\mathbf{K}$ by a set of sentences $B$ can mean either to remove all elements of $B$ from $\mathbf{K}$ (package contractions), or to remove at least one of the elements of $B$ from $\mathbf{K}$ (choice contractions). In the present paper we will only consider the first of those two kinds of operations and we will use the expression multiple contraction to refer to such functions. Furthermore, we will restrict our attention to the case when the sets to be contracted from $\mathbf{K}$ are finite. Hence, throughout this paper whenever we refer to the multiple contraction of a belief set $\mathbf{K}$ by a set of sentences $B$, such set $B$ is assumed to be finite. We will use the expression singleton contraction to designate contractions by a single sentence.

The first constructive model of multiple contractions to be presented was the partial meet multiple contraction in $[13,14]$. The operations of multiple contraction defined within such model are essentially the generalization to the case of contraction by sets of sentences (rather than by a single sentence) of the partial meet (singleton) contraction functions. Nevertheless, a possible worlds semantics has not yet been provided for such generalization.

In this work we aim to provide such possible worlds semantics for the partial meet multiple contractions, inspired in the analogous model for the singleton case proposed by Grove [12] and generalized by Hansson [15].

The paper is organized in the following way: In Section 2 we provide the notation and background needed for the rest of the paper. In Section 3 we provide the possible worlds semantics for the partial meet multiple contractions. The Appendix contains the proofs of all the original results.

\section{Background}

\subsection{Formal Preliminaries}

We will assume a language $\mathcal{L}$ that is closed under truth-functional operations. We shall make use of a consequence operation $C n$ that takes sets of sentences to sets of sentences and which satisfies the following properties:

- inclusion: $A \subseteq \operatorname{Cn}(A)$,

- monotony: if $A \subseteq B$, then $C n(A) \subseteq C n(B)$, 
- idempotence: $\operatorname{Cn}(A)=\operatorname{Cn}(\operatorname{Cn}(A))$,

- supraclassicality: if $\alpha$ can be derived from $A$ by classical truth-functional logic, then $\alpha \in C n(A)$,

- deduction: if $\beta \in C n(A \cup\{\alpha\})$, then $(\alpha \rightarrow \beta) \in C n(A)$,

- compactness: if $\alpha \in C n(A)$, then $\alpha \in C n\left(A^{\prime}\right)$ for some finite subset $A^{\prime}$ of $A$.

We will sometimes use $C n(\alpha)$ for $C n(\{\alpha\}), A \vdash \alpha$ for $\alpha \in C n(A), \vdash \alpha$ for $\alpha \in$ $C n(\emptyset), A \nvdash \alpha$ for $\alpha \notin C n(A), \forall \alpha$ for $\alpha \notin C n(\emptyset)$. The letters $\alpha, \alpha_{i}, \beta, \ldots$ (except for $\gamma$ ) will be used to denote sentences. $\top$ stands for an arbitrary tautology and $\perp$ for an arbitrary contradiction. $A, A_{i}, B, \ldots$ shall denote subsets of sentences of $\mathcal{L} . \mathbf{K}$ is reserved to represent a set of sentences that is closed under logical consequence (i.e. $\mathbf{K}=C n(\mathbf{K}))$ - such a set is called a belief set or theory. The conjunction of all elements of a finite and non-empty set $A$ shall be denoted by $\& A$. We shall denote the set of all maximal consistent subsets of $\mathcal{L}$ by $\mathcal{M}_{\mathcal{L}}$. We will use the expression possible world (or just world) to designate an element of $\mathcal{M}_{\mathcal{L}} . \mathcal{M}, \mathcal{N}_{i}, \mathcal{W}, \ldots$, (except for $\mathcal{L}$ and $\mathcal{P}$ ), shall be used to denote subsets of $\mathcal{M}_{\mathcal{L}}$. Such sets are called propositions. Given a set of sentences $R$, the set consisting of all the possible worlds that contain $R$ is denoted by $\|R\|$. The elements of $\|R\|$ are the $R$-worlds. $\|\varphi\|$ is an abbreviation of $\|\{\varphi\}\|$ and the elements of $\|\varphi\|$ are the $\varphi$-worlds. To any set of possible worlds $\mathcal{V}$ we associate a belief set $T h(\mathcal{V})$ given by $T h(\mathcal{V})=\bigcap \mathcal{V}$ - under the assumption that $\bigcap \emptyset=\mathcal{L}$. $\mathbb{M}, \mathbb{N}_{i}, \mathbb{W}, \ldots$ shall be used to denote subsets of $\mathcal{P}\left(\mathcal{M}_{\mathcal{L}}\right)$.

\subsection{Partial Meet Contractions}

A Partial Meet Contraction function [1, 3] is built upon a selection from the maximal subsets of $\mathbf{K}$ that do not imply the sentence to be contracted. In what follows we present the formal definition of such functions.

Given a belief set $\mathbf{K}$ and a set of sentences $B$, the remainder set of $\mathbf{K}$ by $B$ is the set of maximal subsets of $\mathbf{K}$ that do not imply any element of $B$ and is denoted by $\mathbf{K} \perp B$. Its elements are the remainders (of $\mathbf{K}$, by $B$ ). $\mathbf{K} \perp \alpha$ is an abbreviation of $\mathbf{K} \perp\{\alpha\}$ and is called the remainder set of $\mathbf{K}$ by $\alpha$. It is also convenient to notice here that, according to [2, Observation 2.2], since we are assuming that the consequence operation $C n$ is compact, it holds that $\mathbf{K} \perp B \neq \varnothing$ if and only if $B \cap C n(\emptyset)=\emptyset$.

Definition 2.1 Let $\mathbf{K}$ be a belief set. A selection function for $\mathbf{K}$ is a function $\gamma$ such that for all sentences $\alpha$ : $\emptyset \neq \gamma(\mathbf{K} \perp \alpha) \subseteq \mathbf{K} \perp \alpha$, if $\mathbf{K} \perp \alpha \neq \emptyset$, and $\gamma(\mathbf{K} \perp \alpha)=$ $\{\mathbf{K}\}$, otherwise.

An operation-is a partial meet contraction on $\mathbf{K}$ if and only if there is a selection function $\gamma$ for $\mathbf{K}$ such that for all sentences $\alpha$ : $\mathbf{K}-\alpha=\bigcap \gamma(\mathbf{K} \perp \alpha)$.

Next we present the possible worlds semantics for partial meet contractions. To do that we first notice that propositions (sets of possible worlds) can be used to represent belief sets and sentences. More precisely, a belief set $\mathbf{K}$ can 
be represented by the set $\|\mathbf{K}\|$ and a sentence $\alpha$ can be identified with the set $\|\alpha\|$.

The following result clarifies more formally the above mentioned interrelation between belief sets and sets of possible worlds:

Proposition 2.2 ([4]) Let $\mathbf{H}, \mathbf{K}$ be belief sets and $\mathcal{U}, \mathcal{V}$ be sets of possible worlds. Then:

(i) $\operatorname{Th}(\|\mathbf{K}\|)=\mathbf{K}$.

(ii) If $\mathcal{U} \subseteq \mathcal{V}$, then $\operatorname{Th}(\mathcal{V}) \subseteq T h(\mathcal{U})$.

(iii) If $\mathbf{H} \subseteq \mathbf{K}$, then $\|\mathbf{K}\| \subseteq\|\mathbf{H}\|$.

When considering a propositional approach to represent sentences and belief sets, the contraction of $\mathbf{K}$ by $\alpha$ takes the form of the addition of some $\neg \alpha$ worlds to the set $\|\mathbf{K}\|$. In order to clarify this statement we start by exposing, in the following observation, the very tight interconnection between the sets $\mathbf{K} \perp \varphi$ and $\|\neg \varphi\|$, for some belief set $\mathbf{K}$ and some sentence $\varphi \in \mathbf{K}$ :

Observation 2.3 (Grove's bijection [12, Section 4]) Let $\mathbf{K}$ be a belief set and $\varphi$ be a sentence such that $\varphi \in \mathbf{K}$. Then the function $g$ from $\|\neg \varphi\|$ to $\mathbf{K} \perp \varphi$ defined by $g(W)=T h(\|\mathbf{K}\| \cup\{W\})$, for all $W \in\|\neg \varphi\|$, is a bijection. Moreover $\|g(W)\|=$ $\|\mathbf{K}\| \cup\{W\}$, for all $W \in\|\neg \varphi\|$.

Now we introduce the concept of propositional selection function, which we will use afterwords to present the possible worlds semantics for the partial meet contractions.

Definition 2.4 ([15, Definition 3.65]) Let $\mathcal{M}$ be a proposition. A propositional selection function for $\mathcal{M}$ is a function $f$ such that for all sentences $\alpha$ : (1) $f(\|\alpha\|) \subseteq\|\alpha\|$, (2) If $\|\alpha\| \neq \emptyset$ then $f(\|\alpha\|) \neq \varnothing$ and (3) If $\mathcal{M} \cap\|\alpha\| \neq \emptyset$, then $f(\|\alpha\|)=\mathcal{M} \cap\|\alpha\|$.

We are now in position to present the following observation, which illustrates how the partial meet contractions can be described in terms of possible worlds.

Observation 2.5 ([12], [15, Observation 2.69]) Let $\mathbf{K}$ be a belief set. An operation-on $\mathbf{K}$ is a partial meet contraction if and only if there is a propositional selection function $f$ for $\|\mathbf{K}\|$ such that for all sentences $\alpha$ : $\mathbf{K}-\alpha=$ $\operatorname{Th}(\|\mathbf{K}\| \cup f(\|\neg \alpha\|))$.

\subsection{Partial Meet Multiple Contractions}

Now we recall the definition of the partial meet multiple contractions, which are the generalization of the partial meet contraction functions to the case of contractions by (possibly non-singleton) sets. 
Definition 2.6 (Partial meet multiple contraction $[8,13]$ ) Let $\mathbf{K}$ be a belief set. A package selection function for $\mathbf{K}$ is a function $\gamma$ such that for all sets of sentences $B: \emptyset \neq \gamma(\mathbf{K} \perp B) \subseteq \mathbf{K} \perp B$, if $\mathbf{K} \perp B \neq \emptyset$, and $\gamma(\mathbf{K} \perp B)=\{\mathbf{K}\}$, otherwise.

An operation $\div$ is a partial meet multiple contraction on $\mathbf{K}$ if and only if there is some package selection function $\gamma$ for $\mathbf{K}$, such that for all sets of sentences $B: \mathbf{K} \div B=\bigcap \gamma(\mathbf{K} \perp B)$.

An operation $\div$ on $\mathbf{K}$ is a maxichoice multiple contraction if and only if it is a partial meet multiple contraction generated by a package selection function $\gamma$ such that for all sets $B$, the set $\gamma(\mathbf{K} \perp B)$ has exactly one element. The full meet multiple contraction on $\mathbf{K}$ is the partial meet multiple contraction $\dot{\sim}$ that is generated by the package selection function $\gamma$ such that for all sets $B$, if $\mathbf{K} \perp B \neq \emptyset$, then $\gamma(\mathbf{K} \perp B)=\mathbf{K} \perp B$, i.e., $\mathbf{K} \dot{\sim} B=\bigcap \mathbf{K} \perp B$, if $B \cap C n(\emptyset)=\emptyset$, and $\mathbf{K} \dot{\sim} B=\mathbf{K}$, otherwise.

\section{Possible Worlds Semantics for Partial Meet Multiple Contractions}

In this section our main goal is to obtain the possible worlds semantics for the partial meet multiple contractions. In the case of singleton contraction the possible worlds semantics proposed by Grove [12] and generalized by Hansson [15] is essentially based on the relation between remainder sets (by a single sentence) and sets of possible worlds which is expressed by Grove's bijection (Observation 2.3). Having this in mind, to achieve our goal, we will start by finding out the relation between remainder sets by sets of sentences and sets of possible worlds, which is the generalization of Grove's bijection to the multiple case. Afterwards, by making use of such relation we present the characterization of the partial meet multiple contractions in terms of possible worlds.

\subsection{Possible Worlds Semantics for Remainders}

A partial meet contraction $\mathbf{K}-\alpha$ is defined as the intersection of some of the elements of the remainder set $\mathbf{K} \perp \alpha$, where each element of the remainder set is a maximal subset of $\mathbf{K}$ that does not entail $\alpha$. In the possible worlds model this corresponds to adding to $\|\mathbf{K}\|$ the minimal number of worlds such that $\| \mathbf{K}-$ $\alpha\|\nsubseteq\| \alpha \|$. According Grove's bijection this corresponds simply to adding one and only one $\neg \alpha$-world to $\|\mathbf{K}\|$. By this simple relation, partial meet singleton contraction in terms of possible worlds is defined as $\|\mathbf{K}\|$ plus some (selected) $\neg \alpha$-worlds.

The definition of remainder in multiple contraction is similar to the singleton case, i.e., maximal subsets of $\mathbf{K}$ that do not entail any element of $B$. This corresponds in terms of possible worlds to adding to $\|\mathbf{K}\|$ the minimal number of worlds such that they guarantee that no element of $B$ can be inferred from the remainder set, i.e., $\|\mathbf{K} \div B\| \nsubseteq\left\|\alpha_{i}\right\|$ for all $\alpha_{i} \in B$. More precisely, in what 
follows we will show that if $X \in \mathbf{K} \perp B$, then $X=T h(\|\mathbf{K}\| \cup \mathcal{W})$, for some $\mathcal{W} \subseteq \mathcal{M}_{\mathcal{L}}$ such that, on the one hand $\mathcal{W}$ contains at least one $\neg \alpha_{i}$-world for all $\alpha_{i} \in B \cap \mathbf{K}$ and, on the other hand, if $W \in \mathcal{W}$ then $W$ is the only $\neg \alpha_{j}$-world included in $\mathcal{W}$, for some $\alpha_{j} \in B \cap \mathbf{K}$.

To see that this indeed holds, we start by formally introducing, in the following definition, the set $\mathbb{W}_{\mathbf{K} \perp B}$, composed by all the sets of possible worlds that satisfy the above mentioned conditions.

Definition 3.1 Let $\mathbf{K}$ be a belief set and $B$ be a set of sentences. We denote by $\mathbb{W}_{\mathbf{K} \perp B}$ the subset of $\mathcal{P}\left(\bigcup\left\{\left\|\neg \alpha_{i}\right\|: \alpha_{i} \in B \cap \mathbf{K}\right\}\right)$ such that $\mathcal{W} \in \mathbb{W}_{\mathbf{K} \perp B}$ if and only if:

1. $\mathcal{W} \cap\left\|\neg \alpha_{i}\right\| \neq \emptyset$, for all $\alpha_{i} \in B \cap \mathbf{K}$.

2. If $M \in \mathcal{W}$ then there is some $\alpha_{j} \in B \cap \mathbf{K}$ such that $\mathcal{W} \cap\left\|\neg \alpha_{j}\right\|=\{M\}$.

Although this definition is clear, it is not easy to understand how a remainder set can be "constructed" in terms of possible worlds. The most intuitive approach is to assume that each remainder set corresponds to $\|\mathbf{K}\|$ plus one and only one $\neg \alpha_{i}$-world for each $\alpha_{i} \in B$, which basically correspond to take individual remainders from each member of $B$. However, this is not the case. On Fig. 1 we can see a remainder set which includes more than one $\neg \alpha_{1}$-world. On the other hand, Figs. 2 and 3 show that we cannot define a remainder

Fig. 1 Example of a remainder set for $B=\left\{\alpha_{1}, \alpha_{2}, \alpha_{3}\right\}$ that includes more than one $\neg \alpha_{1}-$ world

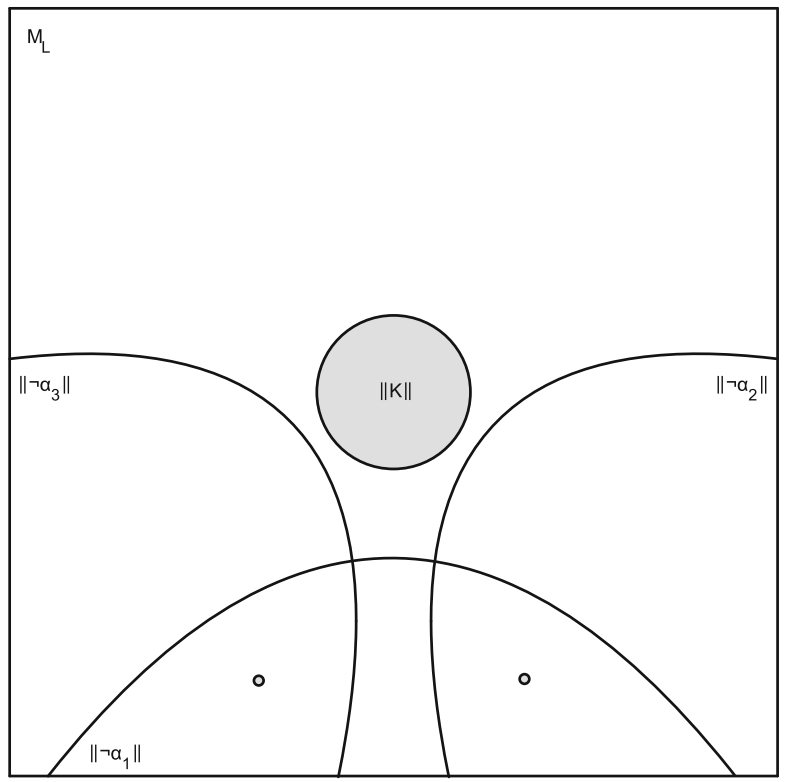


Fig. 2 Example of a remainder set for $B=\left\{\alpha_{1}, \alpha_{2}\right\}$

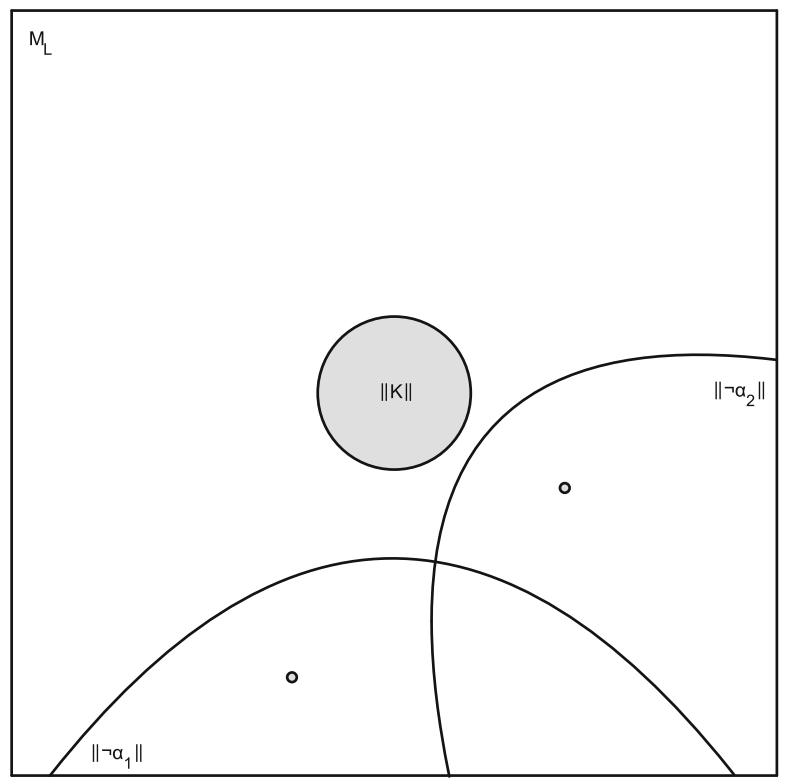

by taking the $\alpha_{i} \in B$ one by one, instead a global strategy is needed. Note that in Fig. 3, if we drop the world in $\left\|\neg \alpha_{2}\right\| \backslash\left\|\neg \alpha_{1}\right\|$ no element of $B$ is recovered.

Fig. 3 Example of a non remainder set for $B=\left\{\alpha_{1}, \alpha_{2}\right\}$

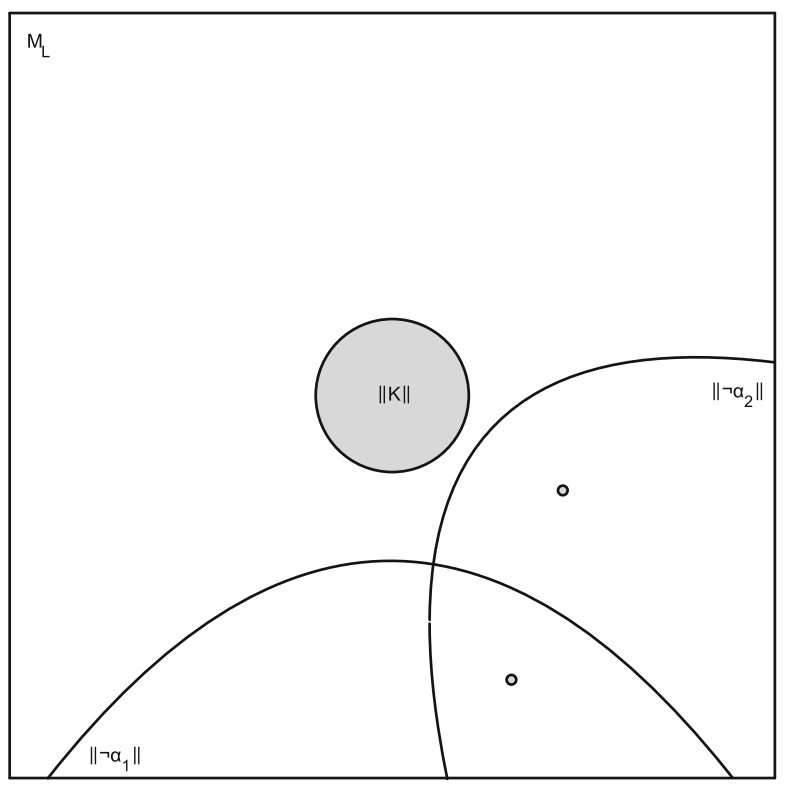

Springer 
Now we investigate the composition of the set $\mathbb{W}_{\mathbf{K} \perp B}$ :

Observation 3.2 Let $\mathbf{K}$ be a belief set and $B$ be a set of sentences. Then the following statements hold:

1. $B \cap C n(\emptyset) \neq \varnothing$ if and only if $\mathbb{W}_{\mathbf{K} \perp B}=\varnothing$.

2. $B \cap \mathbf{K}=\emptyset$ if and only if $\mathbb{W}_{\mathbf{K} \perp B}=\{\emptyset\}$.

3. $B \cap C n(\emptyset)=\emptyset$ and $B \cap \mathbf{K} \neq \varnothing$ if and only if $\mathbb{W}_{\mathbf{K} \perp B} \neq \varnothing$ and $\emptyset \notin \mathbb{W}_{\mathbf{K} \perp B}$.

Notice that according to the above observation (and excluding the limiting cases 1 and 2) the set $\mathbb{W}_{\mathbf{K} \perp B}$ contains at least one non-empty set of worlds, from which it follows that we can use $\mathbb{W}_{\mathbf{K} \perp B}$ in the construction of partial meet multiple contraction by means of a propositional package selection function (as in the singleton case):

Definition 3.3 Let $\mathbf{K}$ be a belief set. A propositional package selection function for $\|\mathbf{K}\|$ is a function $f$ such that for all sets of sentences $B$ : (1) $f\left(\mathbb{W}_{\mathbf{K} \perp B}\right) \subseteq$ $\mathbb{W}_{\mathbf{K} \perp B}$, and (2) if $\mathbb{W}_{\mathbf{K} \perp B} \neq \emptyset$ then $f\left(\mathbb{W}_{\mathbf{K} \perp B}\right) \neq \emptyset$.

In what follows we present the relation between $\mathbb{W}_{\mathbf{K} \perp B}$ and $\mathbf{K} \perp B$. Such relationship will play an analogous role to the one played by Grove's bijection in the singleton case.

Theorem 3.4 Let $\mathbf{K}$ be a belief set and B be a finite set of sentences. Then:

1. If $\mathcal{W} \in \mathbb{W}_{\mathbf{K} \perp B}$ then $X=T h(\|\mathbf{K}\| \cup \mathcal{W}) \in \mathbf{K} \perp B$.

2. If $X \in \mathbf{K} \perp B$ then there is some $\mathcal{W} \in \mathbb{W}_{\mathbf{K} \perp B}$ such that $X=T h(\|\mathbf{K}\| \cup \mathcal{W})$.

The first statement of the theorem means that the sets in $\mathbb{W}_{\mathbf{K} \perp B}$ are minimal sets of worlds in the sense that the addition of each of those sets to $\|\mathbf{K}\|$ corresponds to a maximal subset of $\mathbf{K}$ that does not imply any element of $B$. The second statement yields that each of the remainders can be constructed by means of a set of $\mathbb{W}_{\mathbf{K} \perp B}$.

As an immediate consequence of the above observation we can define $\mathbf{K} \perp B$ in terms of possible worlds:

Corollary 3.5 Let $\mathbf{K}$ be a belief set and $B$ be a finite set of sentences. Then $\mathbf{K} \perp B=\left\{T h(\|\mathbf{K}\| \cup \mathcal{W}): \mathcal{W} \in \mathbb{W}_{\mathbf{K} \perp B}\right\}$.

\subsection{Possible Worlds Semantics for Partial Meet Multiple Contraction}

Having characterized the remainders in terms of possible worlds, our next goal is to obtain the possible worlds semantics for partial meet multiple contractions. As in the singleton case, the lower bound of partial meet multiple contraction would be full meet multiple contraction, hence we will start by 
obtaining the possible worlds semantics for that limiting case of partial meet contractions. Since full meet multiple contraction is given by the intersection of all the element of the remainder set, the corresponding construction in the semantic level is by adding to $\|\mathbf{K}\|$ all the elements of $\bigcup \mathbb{W}_{\mathbf{K} \perp B}$ as it is stated in the following observation:

Observation 3.6 Let $\mathbf{K}$ be a belief set, $B$ a set of sentences and $\dot{\sim}$ be the full meet multiple contraction on $\mathbf{K}$. Then

$$
\mathbf{K} \dot{\sim} B=\bigcap\left(\|\mathbf{K}\| \cup\left(\bigcup \mathbb{W}_{\mathbf{K} \perp B}\right)\right)=\mathbf{K} \cap\left(\bigcap\left(\bigcup \mathbb{W}_{\mathbf{K} \perp B}\right)\right) .
$$

Having seen this, we will now investigate which worlds are included in the set $\bigcup \mathbb{W}_{\mathbf{K} \perp B}$.

Observation 3.7 Let $\mathbf{K}$ be a belief set and B be a set of sentences. Then:

1. $\bigcup \mathbb{W}_{\mathbf{K} \perp B} \subseteq \bigcup\left\{\left\|\neg \alpha_{i}\right\|: \alpha_{i} \in B \cap \mathbf{K}\right\}$.

2. If $B \cap \mathbf{K} \neq \emptyset$ then it may not hold that $\bigcup \mathbb{W}_{\mathbf{K} \perp B}=\bigcup\left\{\left\|\neg \alpha_{i}\right\|: \alpha_{i} \in B \cap \mathbf{K}\right\}$. (Provided that $\mathbf{K} \neq C n(\emptyset))$

3. If $M$ is a world such that (i) $M \in\left\|\neg \alpha_{j}\right\|$ for some $\alpha_{j} \in B \cap \mathbf{K}$, (ii) $M \notin\left\|\neg \alpha_{l}\right\|$ for every $\alpha_{l} \in B \backslash\left\{\alpha_{j}\right\}$, and (iii) there is some $\alpha_{r} \in B \cap \mathbf{K}$ such that $\left\|\neg \alpha_{r}\right\| \subset$ $\left\|\neg \alpha_{j}\right\|$, then $M \notin \bigcup \mathbb{W}_{\mathbf{K} \perp B}$.

The above observation reveals a big difference between the singleton and the multiple case: In singleton contraction, the full meet contraction $\mathbf{K} \sim \alpha$ corresponds to the addition of the whole set $\|\neg \alpha\|$ to $\|\mathbf{K}\|$. However Point 3 of the previous observation clarifies that the full meet multiple contraction does not correspond, in general, to the addition of all the $\neg \alpha_{i}$-worlds to $\|\mathbf{K}\|$.

In order to find out which worlds of the set $\bigcup\left\{\left\|\neg \alpha_{i}\right\|: \alpha_{i} \in B \cap \mathbf{K}\right\}$ are indeed included in $\bigcup \mathbb{W}_{\mathbf{K} \perp B}$, we shall start by finding a way of defining a subset of $\bigcup\left\{\left\|\neg \alpha_{i}\right\|: \alpha_{i} \in B \cap \mathbf{K}\right\}$ that does not contain any world $M$ satisfying (simultaneously) conditions (i)-(iii) mentioned in Point 3 of the previous observation.

Having this in mind, we now notice that, if we consider a subset $B^{\prime}$ of $B$ consisting of those and only those sentences $\alpha_{i}$ in $B$ such that for any sentence $\alpha_{j} \in B$, it holds that $\left\|\neg \alpha_{j}\right\| \not \subset\left\|\neg \alpha_{i}\right\|$, then the elements of the set $\bigcup\left\{\left\|\neg \alpha_{i}\right\|\right.$ : $\alpha_{i} \in B^{\prime} \cap \mathbf{K}$ \} do not simultaneously satisfy the conditions (i)-(iii) mentioned above.

Definition 3.8 (Normalization of a set of sentences) Let $B$ be a set of sentences. The normalization of $B$ is the subset $B_{N} \subseteq B$, defined in the following way:

$$
B_{N}=\left\{\alpha_{i} \in B: \text { For all } \alpha_{j} \in B \text { it holds that }\left\|\neg \alpha_{j}\right\| \not \subset\left\|\neg \alpha_{i}\right\|\right\}
$$


We are now in a position to explicitly indicate which worlds compose the set $\bigcup \mathbb{W}_{\mathbf{K} \perp B}$ :

Observation 3.9 Let $\mathbf{K}$ be a belief set and $B$ be a set of sentences. Then

$$
\bigcup \mathbb{W}_{\mathbf{K} \perp B}=\bigcup\left\{\left\|\neg \alpha_{i}\right\|: \alpha_{i} \in B_{N} \cap \mathbf{K}\right\} .
$$

Combining the identity just presented in the above observation with the result of Observation 3.6 we can obtain a characterization of the full meet multiple contraction: ${ }^{1}$

Observation 3.10 Let $\mathbf{K}$ be a belief set, $B$ be a set of sentences and $\dot{\sim}$ be the operator of full meet multiple contraction on $\mathbf{K}$. Then, under the assumption that $\bigcap \emptyset=\mathcal{L}$, it holds that

$$
\begin{aligned}
\mathbf{K} \dot{*} B & =\bigcap\left(\|\mathbf{K}\| \cup\left(\bigcup\left\{\left\|\neg \alpha_{i}\right\|: \alpha_{i} \in B_{N} \cap \mathbf{K}\right\}\right)\right) \\
& =\mathbf{K} \cap\left(\bigcap\left\{C n\left(\neg \alpha_{i}\right): \alpha_{i} \in B_{N} \cap \mathbf{K}\right\}\right) .
\end{aligned}
$$

Next we introduce one last observation concerning the full meet multiple contraction, that explicitly indicates which worlds compose the set $\|\mathbf{K} \dot{\sim} B\|$ :

Observation 3.11 Let $\mathbf{K}$ be a belief set, $B$ be a set of sentences and $\dot{\sim}$ be the operator of full meet multiple contraction on $\mathbf{K}$. Then

$$
\|\mathbf{K} \dot{\sim} B\|=\|\mathbf{K}\| \cup\left(\bigcup \mathbb{W}_{\mathbf{K} \perp B}\right)=\|\mathbf{K}\| \cup\left(\bigcup\left\{\left\|\neg \alpha_{i}\right\|: \alpha_{i} \in B_{N} \cap \mathbf{K}\right\}\right) .
$$

Now that we have thoroughly exposed the characterization in terms of possible worlds of the full meet multiple contraction, we turn our attention to obtain the possible worlds semantics of the partial meet multiple contractions in general. We start by using Observation 3.11 to obtain a superset of the set $\|\mathbf{K} \div B\|$, where $\div$ is a partial meet multiple contraction on $\mathbf{K}$ :

Observation 3.12 Let $\mathbf{K}$ be a belief set and $B$ be a set of sentences. If an operation $\div$ is a partial meet multiple contraction on $\mathbf{K}$ then

$$
\|\mathbf{K} \div B\| \subseteq\|\mathbf{K}\| \cup\left(\bigcup\left\{\left\|\neg \alpha_{i}\right\|: \alpha_{i} \in B_{N} \cap \mathbf{K}\right\}\right) .
$$

\footnotetext{
${ }^{1}$ Such characterization generalizes the result of [3, Observation 2.1] which states that if $\sim$ is the operator of full meet contraction on a belief set $\mathbf{K}$ then $\mathbf{K} \sim \varphi=\mathbf{K} \cap \operatorname{Cn}(\neg \varphi)$ if $\varphi \in \mathbf{K}$, and $\mathbf{K} \sim$ $\varphi=\mathbf{K}$ if $\varphi \notin \mathbf{K}$. 
Our next goal is to obtain the possible worlds semantics for the partial meet multiple contractions. In order to do that we will present first the following observation:

Observation 3.13 Let $\mathbf{K}$ be a belief set and $B$ and $C$ be sets of sentences. Then

1. $\mathbb{W}_{\mathbf{K} \perp B}=\mathbb{W}_{\mathbf{K} \perp\left(B_{N} \cap \mathbf{K}\right)}$.

2. $\mathbf{K} \perp B=\mathbf{K} \perp C$ iff $\mathbb{W}_{\mathbf{K} \perp B}=\mathbb{W}_{\mathbf{K} \perp C \text {. }}$

The above observation essentially states (through the representation of remainders in terms of possible worlds) that the remainder set of $\mathbf{K}$ by $B$ coincides with the remainder set of $\mathbf{K}$ by $B_{N} \cap \mathbf{K}$. Therefore $\mathbf{K} \div B$ is identical to $\mathbf{K} \div\left(B_{N} \cap \mathbf{K}\right)$ whenever $\div$ is a partial meet multiple contraction. We can now present the main result of the paper, which provides the characterization of partial meet multiple contractions in terms of possible worlds.

Theorem 3.14 Let $\mathbf{K}$ be a belief set. An operation $\div$ is a partial meet multiple contraction on $\mathbf{K}$ if and only if there exists a propositional package selection function $f$ for $\|\mathbf{K}\|$ such that, for any set of sentences $B$ :

$$
\mathbf{K} \div B=\operatorname{Th}\left(\|\mathbf{K}\| \cup\left(\bigcup f\left(\mathbb{W}_{\mathbf{K} \perp B}\right)\right)\right) .
$$

Using the above theorem and Theorem 3.4 it can be easily shown that if $f$ is such that, for any set of sentences $B, f\left(\mathbb{W}_{\mathbf{K} \perp B}\right)$ contains at most one element, then the operation $\div$ defined by $\mathbf{K} \div B=T h\left(\|\mathbf{K}\| \cup\left(\bigcup f\left(\mathbb{W}_{\mathbf{K} \perp B}\right)\right)\right)$ is a maxichoice multiple contraction on $\mathbf{K}$. On the other hand, it is also worth noticing that it follows immediately from Observation 3.6 that the operation $\dot{\sim}$ of full meet multiple contraction on $\mathbf{K}$ is such that $\mathbf{K} \dot{\sim} B=T h\left(\|\mathbf{K}\| \cup\left(\bigcup f\left(\mathbb{W}_{\mathbf{K} \perp B}\right)\right)\right)$, where $f\left(\mathbb{W}_{\mathbf{K} \perp B}\right)=\mathbb{W}_{\mathbf{K} \perp B}$.

\section{Conclusions}

We have presented the possible worlds semantics for the partial meet multiple contraction which generalizes the possible worlds semantics for partial meet (singleton) contraction presented in $[12,15]$.

To be more precise we have shown that every remainder $X \in \mathbf{K} \perp B$ is such that $X=T h(\|\mathbf{K}\| \cup \mathcal{W})$ for some set of possible worlds $\mathcal{W}$ such that (i) $\mathcal{W}$ contains at least one $\neg \alpha_{i}$-world for every $\alpha_{i} \in B \cap \mathbf{K}$ and (ii) for every world in $\mathcal{W}$ there is a sentence $\alpha \in B \cap \mathbf{K}$ for which that one is the only $\neg \alpha$-world in $\mathcal{W}$. Subsequently we have proven that partial meet multiple contractions can be defined by means of intersections of sets of the form $\|\mathbf{K}\| \cup(\cup \mathbb{M})$, where $\mathbb{M}$ consists of a selection of sets of possible worlds that satisfy the above conditions (i) and (ii). 
In particular, we have also characterized in terms of possible worlds the upper and lower bounds of that class of functions, i.e., full meet and maxichoice multiple contractions.

Based on these results, in [5] we introduced a new class of multiple contraction functions - the system of spheres-based multiple contractions - which are a generalization to the case of contraction by (possibly non-singleton) sets of sentences of Grove's system of spheres-based contraction functions.

Acknowledgements We wish to thank the three referees of the JPL and also Jim Delgrande for their valuable comments on a previous version of this paper which have led to improvements in exposition.

\section{Appendix: Proofs}

Previous Lemmas

Lemma 4.1 Let $\mathbf{K}$ be a belief set and $B$ be a set of sentences such that $B \cap$ $\operatorname{Cn}(\emptyset)=\emptyset$. If $X \in \mathbf{K} \perp B$ then $\|X\|=\|\mathbf{K}\| \cup \mathcal{N}$, where $\mathcal{N} \subseteq \mathcal{M}_{\mathcal{L}}$ is such that:

1. $\mathcal{N} \subseteq \bigcup\left\{\left\|\neg \alpha_{i}\right\|: \alpha_{i} \in B\right\}$.

2. $\mathcal{N} \cap\left\|\neg \alpha_{i}\right\| \neq \emptyset$, for all $\alpha_{i} \in B \cap \mathbf{K}$.

Proof If $B \cap \mathbf{K}=\emptyset$ then $\mathbf{K} \perp B=\{\mathbf{K}\}$ and the rest of the proof follows trivially. Now assume $B \cap \mathbf{K} \neq \varnothing$ and let $X \in \mathbf{K} \perp B$.

1. Since $X \subseteq \mathbf{K}$, it follows from Proposition 2.2-(iii) that $\|\mathbf{K}\| \subseteq\|X\|$. Now we prove that $\|X\| \subseteq\|\mathbf{K}\| \cup\left(\bigcup\left\{\left\|\neg \alpha_{i}\right\|: \alpha_{i} \in B\right\}\right)$. Let $M$ be an arbitrary element of $\|X\|$. Assume by redutio that $M \notin\left(\|\mathbf{K}\| \cup\left(\bigcup\left\{\left\|\neg \alpha_{i}\right\|: \alpha_{i} \in B\right\}\right)\right)$, then there is some $\delta \in \mathbf{K}$ such that $\neg \delta \wedge \& B \in M$. Therefore, because $M$ is consistent, it follows that $\delta \vee \neg \& B \notin M$. Then, on the one hand, from $M \in$ $\|X\|$ it follows that $\delta \vee \neg \& B \notin X$ and, on the other hand, since $\delta \in \mathbf{K}$, then $\delta \vee \neg \& B \in \mathbf{K}$. So it holds that $\delta \vee \neg \& B \in \mathbf{K} \backslash X$. Now, since $X \in \mathbf{K} \perp B$, it follows that there is some $\alpha_{j} \in B$ such that $\alpha_{j} \in C n(X \cup\{\delta \vee \neg \& B\})$. Then, by deduction it follows that $(\neg \delta \wedge \& B) \vee \alpha_{j} \in \operatorname{Cn}(X)$. But, from $\alpha_{j} \in B$ it follows that $(\neg \delta \wedge \& B) \vee \alpha_{j} \leftrightarrow \alpha_{j}$. Therefore we obtain that $\alpha_{j} \in \operatorname{Cn}(X)$, which contradicts the fact that $X \in \mathbf{K} \perp B$. Hence we can conclude that $\|X\|=\|\mathbf{K}\| \cup \mathcal{N}$, where $\mathcal{N} \subseteq \bigcup\left\{\left\|\neg \alpha_{i}\right\|: \alpha_{i} \in B\right\}$.

2. Let $\alpha_{i} \in B$ and assume by redutio that $\|X\| \cap\left\|\neg \alpha_{i}\right\|=\emptyset$. Then it follows that $\alpha_{i} \in C n(X)$ which contradicts the fact that $X \in \mathbf{K} \perp B$. Therefore we have that $\|X\| \cap\left\|\neg \alpha_{i}\right\| \neq \emptyset$, for all $\alpha_{i} \in B$, hence $\|\mathcal{N}\| \cap\left\|\neg \alpha_{i}\right\| \neq \emptyset$, for all $\alpha_{i} \in B \cap \mathbf{K}$. 
Lemma 4.2 Let $\mathbf{K}$ be a belief set, $B$ be a finite set of sentences and $\mathcal{N}$ be a subset of $\mathcal{M}_{\mathcal{L}}$. If $\mathcal{N} \cap\left\|\neg \alpha_{i}\right\| \neq \emptyset$, for all $\alpha_{i} \in B \cap \mathbf{K}$, then there is some set $\mathcal{N}^{\prime}$ such that $\mathcal{N}^{\prime} \subseteq \mathcal{N}$ and $\mathcal{N}^{\prime} \in \mathbb{W}_{\mathbf{K} \perp B}{ }^{2}$

Proof If $B \cap \mathbf{K}=\emptyset$ then it is enough to define $\mathcal{N}^{\prime}=\emptyset$ and the required conditions are trivially satisfied. So, we assume $B \cap \mathbf{K} \neq \emptyset$. For each $\alpha_{i} \in B \cap \mathbf{K}$ let $M_{\alpha_{i}}$ be one (arbitrarily chosen) world in $\mathcal{N} \cap\left\|\neg \alpha_{i}\right\|$ (recall that $\mathcal{N} \cap\left\|\neg \alpha_{i}\right\| \neq \emptyset$, for all $\alpha_{i} \in B \cap \mathbf{K}$ ) and consider the set $\mathcal{N}_{0}=\left\{M_{\alpha_{i}}: \alpha_{i} \in B \cap \mathbf{K}\right\}$.

Notice that, since $B$ is finite, $B \cap \mathbf{K}$ also is and, therefore, we may assume without loss of generality that $\mathcal{N}_{0}=\left\{M_{1}, \ldots, M_{n}\right\}$, with $n \geq 1$. Next, for each $m \in\{1, \ldots n\}$, let $\mathcal{N}_{m}$ be the set defined in the following way:

$$
\mathcal{N}_{m}= \begin{cases}\mathcal{N}_{m-1} \backslash\left\{M_{m}\right\} & \text {, if } \mathcal{N}_{m-1} \cap\left\|\neg \alpha_{p}\right\| \neq\left\{M_{m}\right\} \text { for all } \alpha_{p} \in B \cap \mathbf{K} \\ \mathcal{N}_{m-1} & \text { if } \mathcal{N}_{m-1} \cap\left\|\neg \alpha_{q}\right\|=\left\{M_{m}\right\} \text { for some } \alpha_{q} \in B \cap \mathbf{K}\end{cases}
$$

It follows immediately from the above construction that: (1) $\mathcal{N}_{n} \subseteq \mathcal{N} \subseteq$ $\bigcup_{\alpha_{i} \in(B \cap \mathbf{K})}\left\|\neg \alpha_{i}\right\|$, (2) $\mathcal{N}_{n} \cap\left\|\neg \alpha_{k}\right\| \neq \emptyset$, for all $\alpha_{k} \in B \cap \mathbf{K}$ and (3) If $M_{i} \in \mathcal{N}_{n}$ then there is some $\alpha_{q} \in B \cap \mathbf{K}$ such that $\mathcal{N}_{n} \cap\left\|\neg \alpha_{q}\right\|=\left\{M_{i}\right\}$. So according to Definition 3.1, $\mathcal{N}_{n} \in \mathbb{W}_{\mathbf{K} \perp B}$. So, we put $\mathcal{N}^{\prime}=\mathcal{N}_{n}$ and this finishes the proof.

Lemma 4.3 Let $B$ be a set of sentences. If $\alpha_{l} \in B$ and $\alpha_{l} \notin B_{N}$ then there is some $\alpha_{k} \in B_{N}$ such that $\left\|\neg \alpha_{k}\right\| \subset\left\|\neg \alpha_{l}\right\|$.

Proof Let $\alpha_{l} \in B \backslash B_{N}$. It follows from the definition of $B_{N}$ that there is some $\alpha_{s} \in B$ such that $\left\|\neg \alpha_{s}\right\| \subset\left\|\neg \alpha_{l}\right\|$. If $\alpha_{s} \in B_{N}$ this ends the proof. Assume $\alpha_{s} \notin$ $B_{N}$, then using the same arguments, there is some $\alpha_{s_{1}} \in B$ such that $\left\|\neg \alpha_{s_{1}}\right\| \subset$ $\left\|\neg \alpha_{s}\right\|$. Now, on the one hand, if $\alpha_{s_{1}} \in B_{N}$ this ends the proof because under this conditions we have that $\left\|\neg \alpha_{s_{1}}\right\| \subset\left\|\neg \alpha_{l}\right\|$. On the other hand, if $\alpha_{s_{1}} \notin B_{N}$, reasoning in an analogous way we can find a $\alpha_{s_{2}} \in B$ which is related to $\alpha_{s_{1}}$ in precisely in the same way that $\alpha_{s_{1}}$ was related to $\alpha_{s}$ and so on.

Now consider the sequence of sentences $\left(\delta_{n}\right)$ defined in the following way $\delta_{1}=\alpha_{s}, \delta_{2}=\alpha_{s_{1}}, \delta_{3}=\alpha_{s_{2}}, \ldots$, where the next terms of this sequence are the consecutive elements of $B$ obtained using the process just described.

Note that $\left\|\neg \delta_{j}\right\| \nsubseteq\left\|\neg \delta_{i}\right\|$ whenever $j<i$.

Since $B$ is finite there must be some $\delta_{m} \in B$ such that for all $\alpha_{j} \in B$, if $\left\|\neg \alpha_{j}\right\| \subseteq\left\|\neg \delta_{m}\right\|$, then $\left\|\neg \delta_{m}\right\| \subseteq\left\|\neg \alpha_{j}\right\|$, which means that $\delta_{m} \in B_{N}$.

And it follows from the construction of the sequence $\left(\delta_{n}\right)$ that $\left\|\neg \delta_{m}\right\| \subset$ $\left\|\neg \alpha_{l}\right\|$, and this ends the proof.

\footnotetext{
${ }^{2}$ Notice that from $\mathcal{N} \cap\left\|\neg \alpha_{i}\right\| \neq \emptyset$, for all $\alpha_{i} \in B \cap \mathbf{K}$, it follows that $B \cap \operatorname{Cn}(\emptyset)=\emptyset$.
} 
Lemma 4.4 Let $\mathbf{K}$ be a belief set, $B$ be a set of sentences such that $B \cap C n(\emptyset)=\emptyset$ and $f:\{\|\alpha\|: \alpha \in \mathcal{L}$ and $\forall \neg \alpha\} \rightarrow \mathcal{P}\left(\mathcal{M}_{\mathcal{L}}\right)$ be a function such that if $\alpha \in \mathcal{L}$ and $\forall \neg \alpha$ then $\emptyset \neq f(\|\alpha\|) \subseteq\|\alpha\|$. If $B_{f} \subseteq B$ is such that:

(i) If $\alpha_{j} \in B_{f}$ then $f\left(\left\|\neg \alpha_{k}\right\|\right)=f\left(\left\|\neg \alpha_{j}\right\|\right)$ or $f\left(\left\|\neg \alpha_{k}\right\|\right) \not \subset\left\|\neg \alpha_{j}\right\|$, for all $\alpha_{k} \in$ $B_{f}$,

(ii) For all $\alpha_{l} \in B \cap \mathbf{K}$ there is some $\alpha_{m} \in B_{f} \cap \mathbf{K}$ such that $f\left(\left\|\neg \alpha_{m}\right\|\right) \subseteq$ $\left\|\neg \alpha_{l}\right\|$,

then

1. For every world $M \in \bigcup\left\{f\left(\left\|\neg \alpha_{i}\right\|\right): \alpha_{i} \in B_{f} \cap \mathbf{K}\right\}$ there is some subset $\mathcal{W}_{M} \subseteq \bigcup\left\{f\left(\left\|\neg \alpha_{i}\right\|\right): \alpha_{i} \in B_{f} \cap \mathbf{K}\right\}$, such that $M \in \mathcal{W}_{M}$ and $\mathcal{W}_{M} \in \mathbb{W}_{\mathbf{K} \perp B}$.

2. $\left\{\mathcal{W} \in \mathbb{W}_{\mathbf{K} \perp B}: \mathcal{W} \subseteq \bigcup\left\{f\left(\left\|\neg \alpha_{i}\right\|\right): \alpha_{i} \in B_{f} \cap \mathbf{K}\right\}\right\} \neq \emptyset$ and $\bigcup\left\{\mathcal{W} \in \mathbb{W}_{\mathbf{K} \perp B}\right.$ : $\left.\mathcal{W} \subseteq \bigcup\left\{f\left(\left\|\neg \alpha_{i}\right\|\right): \alpha_{i} \in B_{f} \cap \mathbf{K}\right\}\right\}=\bigcup\left\{f\left(\left\|\neg \alpha_{i}\right\|\right): \alpha_{i} \in B_{f} \cap \mathbf{K}\right\}$.

Proof Let $B_{f} \subseteq B$ be such that conditions (i) and (ii) are satisfied.

1. If $B \cap \mathbf{K}=\emptyset$ then $B_{f} \cap \mathbf{K}=\emptyset$ and 1 . is vacuously true. Let $B \cap \mathbf{K} \neq \emptyset$. Let $\alpha_{j}$ be an arbitrary element of $B_{f} \cap \mathbf{K}$ and $M$ be an arbitrary element of $f\left(\left\|\neg \alpha_{j}\right\|\right)$. First we prove that if $\alpha_{l} \in B \cap \mathbf{K} \cap M$ then $\bigcup\left\{f\left(\left\|\neg \alpha_{i}\right\|\right): \alpha_{i} \in\right.$ $\left.B_{f} \cap \mathbf{K}\right\} \cap\left\|\alpha_{j}\right\| \cap\left\|\neg \alpha_{l}\right\| \neq \emptyset$. Let $\alpha_{l}$ be an arbitrary element of $B \cap \mathbf{K} \cap$ $M$. Then from (ii), it follows that there is some $\alpha_{m} \in B_{f} \cap \mathbf{K}$ such that $f\left(\left\|\neg \alpha_{m}\right\|\right) \subseteq\left\|\neg \alpha_{l}\right\|$.

Furthermore, in these conditions, it holds that $f\left(\left\|\neg \alpha_{m}\right\|\right) \nsubseteq\left\|\neg \alpha_{j}\right\|$. To prove this we will show that (a) $f\left(\left\|\neg \alpha_{m}\right\|\right) \neq\left\|\neg \alpha_{j}\right\|$ and (b) $f\left(\left\|\neg \alpha_{m}\right\|\right) \not \subset$ $\left\|\neg \alpha_{j}\right\|$.

(a) Since $\alpha_{l} \in M$ it follows that $M \notin\left\|\neg \alpha_{l}\right\|$ and because $f\left(\left\|\neg \alpha_{m}\right\|\right) \subseteq$ $\left\|\neg \alpha_{l}\right\|$, we obtain that $M \notin f\left(\left\|\neg \alpha_{m}\right\|\right)$. On the other hand, from $M \in$ $f\left(\left\|\neg \alpha_{j}\right\|\right)$ and $f\left(\left\|\neg \alpha_{j}\right\|\right) \subseteq\left\|\neg \alpha_{j}\right\|$ it follows that $M \in\left\|\neg \alpha_{j}\right\|$ and we are done.

(b) Due to $\alpha_{m} \in B_{f}$, according to (i), it holds that $f\left(\left\|\neg \alpha_{m}\right\|\right)=f\left(\left\|\neg \alpha_{j}\right\|\right)$ or $f\left(\left\|\neg \alpha_{m}\right\|\right) \not \subset\left\|\neg \alpha_{j}\right\|$. On the other hand, since $M \in f\left(\left\|\neg \alpha_{j}\right\|\right)$, from $M \notin f\left(\left\|\neg \alpha_{m}\right\|\right)$ (already proven above) it follows that $f\left(\left\|\neg \alpha_{m}\right\|\right) \neq$ $f\left(\left\|\neg \alpha_{j}\right\|\right)$. Therefore $f\left(\left\|\neg \alpha_{m}\right\|\right) \not \subset\left\|\neg \alpha_{j}\right\|$.

Hence, if $\alpha_{l} \in B \cap \mathbf{K} \cap M$ it holds that $\bigcup\left\{f\left(\left\|\neg \alpha_{i}\right\|\right): \alpha_{i} \in B_{f} \cap \mathbf{K}\right\} \cap\left\|\alpha_{j}\right\| \cap$ $\left\|\neg \alpha_{l}\right\| \neq \emptyset$.

Let $\mathcal{W}$ be the set defined by $\mathcal{W}=\{M\} \cup\left\{M_{\alpha_{l}}: \alpha_{l} \in B \cap \mathbf{K} \cap M\right\}$, where for each $\alpha_{l} \in B \cap \mathbf{K} \cap M, M_{\alpha_{l}}$ is an arbitrary element of $\bigcup\left\{f\left(\left\|\neg \alpha_{i}\right\|\right): \alpha_{i} \in\right.$ $\left.B_{f} \cap \mathbf{K}\right\} \cap\left\|\alpha_{j}\right\| \cap\left\|\neg \alpha_{l}\right\|$. Since $\mathcal{W} \subseteq \mathcal{M}_{\mathcal{L}}$ is such that $\mathcal{W} \cap\left\|\neg \alpha_{k}\right\| \neq \emptyset$ for all $\alpha_{k} \in B \cap \mathbf{K}$, it follows immediately from Lemma 4.2 that there is some set $\mathcal{W}^{\prime}$ such that $\mathcal{W}^{\prime} \subseteq \mathcal{W}$ and $\mathcal{W}^{\prime} \in \mathbb{W}_{\mathbf{K} \perp B}$.

Furthermore, $\mathcal{W}^{\prime} \subseteq \bigcup\left\{f\left(\left\|\neg \alpha_{i}\right\|\right): \alpha_{i} \in B_{f} \cap \mathbf{K}\right\}$ and, due to $\mathcal{W} \cap\left\|\neg \alpha_{j}\right\|=$ $\{M\}$, recalling that $\mathcal{W}^{\prime} \cap\left\|\neg \alpha_{i}\right\| \neq \emptyset$, for all $\alpha_{i} \in B \cap \mathbf{K}$ (according to Definition 3.1), from $\mathcal{W}^{\prime} \subseteq \mathcal{W}$ we can conclude that $M \in \mathcal{W}^{\prime}$. 
Hence, if we define $\mathcal{W}_{M}=\mathcal{W}^{\prime}$ then $\mathcal{W}_{M} \subseteq \bigcup\left\{f\left(\left\|\neg \alpha_{i}\right\|\right): \alpha_{i} \in B_{f} \cap \mathbf{K}\right\}$, $M \in \mathcal{W}_{M}$ and $\mathcal{W}_{M} \in \mathbb{W}_{\mathbf{K} \perp B}$, as required.

2. Case $1, B \cap \mathbf{K}=\emptyset$. Then, according to Observation 3.2-2., $\mathbb{W}_{\mathbf{K} \perp B}=\{\emptyset\}$. Hence, $\left\{\mathcal{W} \in \mathbb{W}_{\mathbf{K} \perp B}: \mathcal{W} \subseteq \bigcup\left\{f\left(\left\|\neg \alpha_{i}\right\|\right): \alpha_{i} \in B_{f} \cap \mathbf{K}\right\}\right\}=\{\emptyset\} \neq \emptyset$.

On the other hand, since $B_{f} \subseteq B$, it holds that $B_{f} \cap \mathbf{K}=\emptyset$ and, consequently, $\bigcup\left\{f\left(\left\|\neg \alpha_{i}\right\|\right): \alpha_{i} \in B_{f} \cap \mathbf{K}\right\}=\bigcup \emptyset=\emptyset$ and the rest is trivial.

Case $2, B \cap \mathbf{K} \neq \emptyset$. Then, from (ii) we can conclude that $B f \cap \mathbf{K} \neq \emptyset$ and it follows immediately from 1 (already proven above) that $\left\{\mathcal{W} \in \mathbb{W}_{\mathbf{K} \perp B}\right.$ : $\left.\mathcal{W} \subseteq \bigcup\left\{f\left(\left\|\neg \alpha_{i}\right\|\right): \alpha_{i} \in B_{f} \cap \mathbf{K}\right\}\right\} \neq \emptyset$. Finally it follows immediately from 1. that $\bigcup\left\{\mathcal{W} \in \mathbb{W}_{\mathbf{K} \perp B}: \mathcal{W} \subseteq \bigcup\left\{f\left(\left\|\neg \alpha_{i}\right\|\right): \alpha_{i} \in B_{f} \cap \mathbf{K}\right\}\right\}=\bigcup\left\{f\left(\left\|\neg \alpha_{i}\right\|\right)\right.$ : $\left.\alpha_{i} \in B_{f} \cap \mathbf{K}\right\}$.

Lemma 4.5 Let $\mathbf{K}$ be a belief set and $B$ be a set of sentences such that $B \cap$ $\operatorname{Cn}(\emptyset)=\emptyset$. Then $\bigcup\left\{\mathcal{W} \in \mathbb{W}_{\mathbf{K} \perp B}: \mathcal{W} \subseteq \bigcup\left\{\left\|\neg \alpha_{i}\right\|: \alpha_{i} \in B_{N} \cap \mathbf{K}\right\}\right\}=\bigcup\left\{\left\|\neg \alpha_{i}\right\|:\right.$ $\left.\alpha_{i} \in B_{N} \cap \mathbf{K}\right\}$.

Proof We will prove this lemma using Lemma 4.4-2. Considering the function $f:\{\|\alpha\|: \alpha \in \mathcal{L}$ and $\forall \neg \neg \alpha\} \rightarrow \mathcal{P}\left(\mathcal{M}_{\mathcal{L}}\right)$ defined by $f(\|\alpha\|)=\|\alpha\|$ and $B_{f}=$ $B_{N}$ where $B_{N}$ is the normalization of $B$. Then it obviously holds that if $\alpha \in \mathcal{L}$ and $\forall \neg \alpha$ then $\emptyset \neq f(\|\alpha\|) \subseteq\|\alpha\|$. In order to finish the proof we prove that both conditions (i) and (ii) of Lemma 4.4 are satisfied. Condition (i) follows immediately from Definition 3.8. For (ii) let $\alpha_{l}$ be an arbitrary element of $B \cap \mathbf{K}$, we must show that there is some $\alpha_{m} \in B_{N} \cap \mathbf{K}$ such that $\left\|\neg \alpha_{m}\right\| \subseteq$ $\left\|\neg \alpha_{l}\right\|$ (since $\left.f\left(\left\|\neg \alpha_{m}\right\|\right)=\left\|\neg \alpha_{m}\right\|\right)$. To prove this we consider two cases:

Case $1 \quad \alpha_{l} \in B_{N}$. Then $\alpha_{l} \in B_{N} \cap \mathbf{K}$ and $\left\|\neg \alpha_{l}\right\| \subseteq\left\|\neg \alpha_{l}\right\|$, and we are done.

Case $2 \alpha_{l} \notin B_{N}$. Then, it follows from Lemma 4.3 that there is some $\alpha_{m} \in$ $B_{N}$ such that $\left\|\neg \alpha_{m}\right\| \subset\left\|\neg \alpha_{l}\right\|$. On the other hand, from $\alpha_{l} \in \mathbf{K}$ and $\left\|\neg \alpha_{m}\right\| \subset\left\|\neg \alpha_{l}\right\|$ we can conclude that $\alpha_{m} \in \mathbf{K}$. Hence, we have that $\alpha_{m} \in B_{N} \cap \mathbf{K}$ and $\left\|\neg \alpha_{m}\right\| \subseteq\left\|\neg \alpha_{l}\right\|$, as required and this finishes the proof.

Lemma 4.6 Let $\mathbf{K}$ be a belief set and $G$ and $H$ be sets of sentences. If every subset $X$ of $\mathbf{K}$ implies some element of $G$ if and only if $X$ implies some element of $H$, then for all $\beta_{i} \in G_{N} \cap \mathbf{K}$ there is some $\varsigma_{i} \in H_{N} \cap \mathbf{K}$ such that $\left\|\beta_{i}\right\|=\left\|\varsigma_{i}\right\|$.

Proof Assume that every subset $X$ of $\mathbf{K}$ implies some element of $G$ if and only if $X$ implies some element of $H$ and let $\beta_{i} \in G_{N} \cap \mathbf{K}$. We will start by showing that there is an element of $H_{N} \cap \mathbf{K}$ which is logically equivalent to $\beta_{i}$. Since $\left\{\beta_{i}\right\} \subseteq \mathbf{K}$ and $\left\{\beta_{i}\right\} \vdash \beta_{i} \in G$, it follows from the hypothesis that there is some $\varsigma_{i} \in H$ such that $\left\{\beta_{i}\right\} \vdash \varsigma_{i}$. Then, by deduction we have that $\vdash \beta_{i} \rightarrow \varsigma_{i}$ which is equivalent to $\left\|\neg \varsigma_{i}\right\| \subseteq\left\|\neg \beta_{i}\right\|$. Since $\mathbf{K}$ is a belief set, we can conclude that $\varsigma_{i} \in \mathbf{K}$, hence, proceeding analogously, we can conclude that there is some $\beta_{j} \in G$ such that $\left\|\neg \beta_{j}\right\| \subseteq\left\|\neg \varsigma_{i}\right\|$. Then we have that $\left\|\neg \beta_{j}\right\| \subseteq\left\|\neg \beta_{i}\right\|$, and from 
the fact that $\beta_{i} \in G_{N}$ we can conclude that $\left\|\neg \beta_{j}\right\|=\left\|\neg \beta_{i}\right\|$. So, it holds that $\left\|\neg \beta_{i}\right\|=\left\|\neg \varsigma_{i}\right\|$.

It remains to prove that $\varsigma_{i} \in H_{N} \cap \mathbf{K}$. We already have that $\varsigma_{i} \in H \cap \mathbf{K}$. To show that $\varsigma_{i} \in H_{N}$ we must prove that for all $\varsigma_{j} \in H$ it holds that if $\left\|\neg \varsigma_{j}\right\| \subseteq\left\|\neg \varsigma_{i}\right\|$ then $\left\|\neg \varsigma_{i}\right\| \subseteq\left\|\neg \varsigma_{j}\right\|$. So, let $\varsigma_{j} \in H$ be such that $\left\|\neg \varsigma_{j}\right\| \subseteq\left\|\neg \varsigma_{i}\right\|$. Since $\left\|\neg \beta_{i}\right\|=\left\|\neg \varsigma_{i}\right\|$, it follows that $\left\|\neg \varsigma_{j}\right\| \subseteq\left\|\neg \beta_{i}\right\|$. Reasoning as above we can conclude that there is some $\beta_{l} \in G$ such that $\left\|\neg \beta_{l}\right\| \subseteq\left\|\neg \varsigma_{j}\right\|$. Then we have that $\left\|\neg \beta_{l}\right\| \subseteq\left\|\neg \beta_{i}\right\|$, and again from the fact that $\beta_{i} \in G_{N}$ we obtain that $\left\|\neg \beta_{l}\right\|=\left\|\neg \beta_{i}\right\|$, which allows us to conclude that $\left\|\neg \varsigma_{i}\right\| \subseteq\left\|\neg \varsigma_{j}\right\|$ as required.

\section{Proofs}

Proof of Observation 3.2 In order to prove that the statements 1.-3. are satisfied it is enough to show that (i) if $B \cap C n(\emptyset) \neq \varnothing$ then $\mathbb{W}_{\mathbf{K} \perp B}=\emptyset$, (ii) if $B \cap \mathbf{K}=\emptyset$ then $\mathbb{W}_{\mathbf{K} \perp B}=\{\emptyset\}$, and (iii) if $B \cap C n(\emptyset)=\emptyset$ and $B \cap \mathbf{K} \neq \emptyset$ then $\mathbb{W}_{\mathbf{K} \perp B} \neq \varnothing$ and $\emptyset \notin \mathbb{W}_{\mathbf{K} \perp B}$.

(i) Let $B \cap C n(\emptyset) \neq \emptyset$. We will prove that $\mathbb{W}_{\mathbf{K} \perp B}=\emptyset$. Let $\alpha_{j} \in B \cap C n(\emptyset)$. Then $\alpha_{j} \in B \cap \mathbf{K}$ and $\left\|\neg \alpha_{j}\right\|=\emptyset$. Therefore, there is no $\mathcal{W}$ such that $\mathcal{W} \cap$ $\left\|\neg \alpha_{i}\right\| \neq \emptyset$, for all $\alpha_{i} \in B \cap \mathbf{K}$. Hence $\mathbb{W}_{\mathbf{K} \perp B}=\emptyset$.

(ii) Let $B \cap \mathbf{K}=\emptyset$. Then it follows that $\left\{\left\|\neg \alpha_{i}\right\|: \alpha_{i} \in B \cap \mathbf{K}\right\}=\emptyset$ and then, by Definition 3.1, the set $\mathbb{W}_{\mathbf{K} \perp B}$ is such that $\mathbb{W}_{\mathbf{K} \perp B} \subseteq \mathcal{P}(\emptyset)=\{\emptyset\}$. Therefore either $\mathbb{W}_{\mathbf{K} \perp B}=\emptyset$ or $\mathbb{W}_{\mathbf{K} \perp B}=\{\emptyset\}$. Given that $B \cap \mathbf{K}=\emptyset$, we have trivially that $1 . \emptyset \cap\left\|\neg \alpha_{i}\right\| \neq \emptyset$, for all $\alpha_{i} \in B \cap \mathbf{K}$; and 2. If $M \in \emptyset$ then there is some $\alpha_{j} \in B \cap \mathbf{K}$ such that $\emptyset \cap\left\|\neg \alpha_{j}\right\|=M$. Hence, according to Definition 3.1, $\varnothing \in \mathbb{W}_{\mathbf{K} \perp B}$.

(iii) Let $B \cap C n(\emptyset)=\emptyset$ and $B \cap \mathbf{K} \neq \emptyset$. Then for every $\alpha_{i} \in B \cap \mathbf{K}$ we have that $\left\|\neg \alpha_{i}\right\| \neq \emptyset$. Let $M_{\alpha_{i}}$ be one (arbitrarily chosen) $\neg \alpha_{i}$-world and consider the set $\mathcal{N}=\left\{M_{\alpha_{i}}: \alpha_{i} \in B \cap \mathbf{K}\right\}$. It follows immediately from Lemma 4.2 that there is some set $\mathcal{N}^{\prime}$ such that $\mathcal{N}^{\prime} \subseteq \mathcal{N}$ and $\mathcal{N}^{\prime} \in \mathbb{W}_{\mathbf{K} \perp B}$. Hence $\mathbb{W}_{\mathbf{K} \perp B} \neq \emptyset$. Let $\alpha_{j}$ be an arbitrary sentence of $B \cap \mathbf{K}$. Then, since $\emptyset \cap\left\|\neg \alpha_{j}\right\|=\varnothing$, it follows from Definition 3.1 that $\emptyset \notin \mathbb{W}_{\mathbf{K} \perp B}$.

\section{Proof of Theorem 3.4}

1. We will consider three cases separately:

Case a $B \cap C n(\emptyset) \neq \varnothing$. Then, according to Observation 3.2-1., it holds that $\mathbb{W}_{\mathbf{K} \perp B}=\emptyset$ and we are done.

Case b $B \cap \mathbf{K}=\emptyset$. Then, by Observation 3.2-2., we have that $\mathbb{W}_{\mathbf{K} \perp B}=$ $\{\emptyset\}$. On the other hand $\mathbf{K} \perp B=\{\mathbf{K}\}$. Hence, it follows immediately from the fact that $T h(\|\mathbf{K}\| \cup \emptyset)=T h(\|\mathbf{K}\|)=\mathbf{K}$ (by Proposition 2.2-(i)) that if $\mathcal{W} \in \mathbb{W}_{\mathbf{K} \perp B}$ then $T h(\|\mathbf{K}\| \cup \mathcal{W}) \in \mathbf{K} \perp B$.

Case c $B \cap C n(\emptyset)=\emptyset$ and $B \cap \mathbf{K} \neq \emptyset$. Then, by Observation 3.2-3., $\mathbb{W}_{\mathbf{K} \perp B} \neq \emptyset$ and $\emptyset \notin \mathbb{W}_{\mathbf{K} \perp B}$. Let $\mathcal{W} \in \mathbb{W}_{\mathbf{K} \perp B} . \quad T h(\|\mathbf{K}\| \cup \mathcal{W})=$ $\bigcap(\|\mathbf{K}\| \cup \mathcal{W})=(\bigcap\|\mathbf{K}\|) \cap(\bigcap \mathcal{W})=\mathbf{K} \cap(\bigcap \mathcal{W})$. Hence, we have 
to prove that (i) $\mathbf{K} \cap(\cap \mathcal{W}) \subseteq \mathbf{K}$; (ii) $C n(\mathbf{K} \cap(\cap \mathcal{W})) \cap B=\emptyset$ and (iii) if $\beta \in \mathbf{K} \backslash(\mathbf{K} \cap(\cap \mathcal{W}))$ then $C n((\mathbf{K} \cap(\cap \mathcal{W})) \cup\{\beta\}) \cap$ $B \neq \varnothing$.

Condition (i) is trivially satisfied. Regarding (ii), let $\alpha_{i}$ be an arbitrary element of $B$, we will show that $\alpha_{i} \notin C n(\mathbf{K} \cap(\bigcap \mathcal{W}))$. If $\alpha_{i} \in B \backslash \mathbf{K}$, it is obvious that $\alpha_{i} \notin C n(\mathbf{K} \cap(\cap \mathcal{W}))$. If $\alpha_{i} \in B \cap \mathbf{K}$, then, according to Definition 3.1, there is some world $M \in \mathcal{W}$ such that $M \in\left\|\neg \alpha_{i}\right\|$. Therefore $\alpha_{i} \notin M$. Hence, since $\mathbf{K} \cap(\cap \mathcal{W}) \subseteq M, \alpha_{i} \notin C n(\mathbf{K} \cap(\cap \mathcal{W}))$. Finally, in order to prove (iii), let $\beta \in \mathbf{K} \backslash(\mathbf{K} \cap(\cap \mathcal{W}))$. Hence there is some $M \in$ $\mathcal{W}$ such that $\beta \notin M$. Therefore, because $M \in \mathcal{M}_{\mathcal{L}}$, it holds that $\neg \beta \in$ $M$. Since $M \in \mathcal{W}$, according to Definition 3.1, there is some $\alpha_{j} \in B \cap \mathbf{K}$ such that $\mathcal{W} \cap\left\|\neg \alpha_{j}\right\|=\{M\}$. Now, from $\neg \beta \in M, \alpha_{j} \in \mathbf{K}$ and $\alpha_{j} \in W_{i}$, for every $W_{i} \in \mathcal{W} \backslash M$, it follows that $\neg \beta \vee \alpha_{j} \in(\mathbf{K} \cap(\cap \mathcal{W}))$. Therefore $\alpha_{j} \in \operatorname{Cn}((\mathbf{K} \cap(\cap \mathcal{W})) \cup\{\beta\})$, and then $\operatorname{Cn}((\mathbf{K} \cap(\cap \mathcal{W})) \cup\{\beta\}) \cap B \neq \varnothing$ as required.

2. We will divide the proof in cases:

Case a $B \cap C n(\emptyset) \neq \emptyset$. Then $\mathbf{K} \perp B=\emptyset$ and we are done.

Case b $\quad B \cap \mathbf{K}=\emptyset$. Let $X \in \mathbf{K} \perp B$. Then $X=\mathbf{K}$ (because $\mathbf{K} \perp B=\{\mathbf{K}\}$ ). According to Observation 3.2-2. it holds that $\mathbb{W}_{\mathbf{K} \perp B}=\{\emptyset\}$. Observing that $T h(\|\mathbf{K}\| \cup \emptyset)=T h(\|\mathbf{K}\|)=\mathbf{K}$ (by Proposition 2.2(i)), we can conclude that $\mathcal{W}=\emptyset$ is such that $\mathcal{W} \in \mathbb{W}_{\mathbf{K} \perp B}$ and $X=T h(\|\mathbf{K}\| \cup \mathcal{W})$, as required.

Case c $B \cap C n(\emptyset)=\emptyset$ and $B \cap \mathbf{K} \neq \emptyset$. Let $X \in \mathbf{K} \perp B$. It follows from Lemma 4.1 that $\|X\|=\|\mathbf{K}\| \cup \mathcal{N}$, where $\mathcal{N} \subseteq \mathcal{M}_{\mathcal{L}}$ is such that $\mathcal{N} \cap\left\|\neg \alpha_{i}\right\| \neq \emptyset$, for all $\alpha_{i} \in B \cap \mathbf{K}$. Then, since it follows from Proposition 2.2-(i) that $X=T h(\|X\|)$, we can conclude that $X=$ $\operatorname{Th}(\|\mathbf{K}\| \cup \mathcal{N})$.

On the other hand, in the above conditions, according to Lemma 4.2, there is some set $\mathcal{N}^{\prime}$ such that $\mathcal{N}^{\prime} \subseteq \mathcal{N}$ and $\mathcal{N}^{\prime} \in \mathbb{W}_{\mathbf{K} \perp B}$.

Let $X^{\prime}=T h\left(\|\mathbf{K}\| \cup \mathcal{N}^{\prime}\right)$. To finish the proof it is enough to show that $X=$ $X^{\prime}$. Now, to see that this is indeed the case, we start by observing that, since $\|\mathbf{K}\| \cup \mathcal{N}^{\prime} \subseteq\|\mathbf{K}\| \cup \mathcal{N}$, it follows from Proposition 2.2-(ii) that $X=$ $T h(\|\mathbf{K}\| \cup \mathcal{N}) \subseteq T h\left(\|\mathbf{K}\| \cup \mathcal{N}^{\prime}\right)=X^{\prime}$. Furthermore, according to point 1 . proven above, because $\mathcal{N}^{\prime} \in \mathbb{W}_{\mathbf{K} \perp B}$, we also have that $X^{\prime} \in \mathbf{K} \perp B$. Hence, in particular it holds that $X^{\prime} \subseteq \mathbf{K}$ and $C n\left(X^{\prime}\right) \cap B=\emptyset$. From this and the fact that $X \in \mathbf{K} \perp B$ it follows that $X \not \subset X^{\prime}$. Therefore, since $X \subseteq X^{\prime}$, we can conclude that $X=X^{\prime}$, and this finishes the proof.

Proof of Observation 3.6 If $B \cap C n(\emptyset)=\emptyset$, according to Corollary 3.5 it follows that $\mathbf{K} \dot{\sim} B=\bigcap\left\{T h(\|\mathbf{K}\| \cup \mathcal{W}): \mathcal{W} \in \mathbb{W}_{\mathbf{K} \perp B}\right\}=\bigcap\{\bigcap(\|\mathbf{K}\| \cup \mathcal{W})$ : $\left.\mathcal{W} \in \mathbb{W}_{\mathbf{K} \perp B}\right\}=\bigcap\left(\|\mathbf{K}\| \cup\left(\bigcup \mathbb{W}_{\mathbf{K} \perp B}\right)\right)=(\cap\|\mathbf{K}\|) \cap\left(\cap\left(\bigcup \mathbb{W}_{\mathbf{K} \perp B}\right)\right)=$ $\mathbf{K} \cap\left(\cap\left(\bigcup \mathbb{W}_{\mathbf{K} \perp B}\right)\right)$.

Let $B \cap C n(\emptyset) \neq \emptyset$. Then, on the one hand, $\mathbf{K} \dot{\sim} B=\mathbf{K}$ and, on the other hand, $\mathbb{W}_{\mathbf{K} \perp B}=\emptyset$ (according to Observation 3.2-1.) and, consequently, 
$\bigcup \mathbb{W}_{\mathbf{K} \perp B}=\emptyset$. Hence we only need to show that $\mathbf{K}=\bigcap(\|\mathbf{K}\| \cup \emptyset)$ and $\mathbf{K}=$ $\mathbf{K} \cap(\bigcap \emptyset)$. But, the former identity follows immediately from Proposition 2.2(i), and the latter equality is an obvious consequence of our assumption that $\operatorname{Th}(\emptyset)=\bigcap \emptyset=\mathcal{L}$.

\section{Proof of Observation 3.7}

1. According to Definition 3.1, we have that $\mathbb{W}_{\mathbf{K} \perp B} \subseteq \mathcal{P}\left(\bigcup\left\{\left\|\neg \alpha_{i}\right\|\right.\right.$ : $\left.\alpha_{i} \in B \cap \mathbf{K}\right\}$ ), which implies immediately that $\bigcup \mathbb{W}_{\mathbf{K} \perp B} \subseteq \bigcup\left\{\left\|\neg \alpha_{i}\right\|: \alpha_{i} \in\right.$ $B \cap \mathbf{K}\}$.

2. We will show a counterexample: Let $\alpha_{1}, \alpha_{2}, \alpha_{3}$ be three sentences such that $\alpha_{1}, \alpha_{2}, \alpha_{3} \in \mathbf{K} \backslash \operatorname{Cn}(\emptyset)$. Furthermore, assume that $\left\|\neg \alpha_{2}\right\| \subset\left\|\neg \alpha_{1}\right\|$, $\left\|\neg \alpha_{2}\right\| \not \subset\left\|\neg \alpha_{3}\right\|,\left\|\neg \alpha_{3}\right\| \not \subset\left\|\neg \alpha_{2}\right\|$ and $\left\|\neg \alpha_{1}\right\| \backslash\left(\left\|\neg \alpha_{2}\right\| \cup\left\|\neg \alpha_{3}\right\|\right) \neq \emptyset$ and let $M$ be an arbitrary element of $\left\|\neg \alpha_{1}\right\| \backslash\left(\left\|\neg \alpha_{2}\right\| \cup\left\|\neg \alpha_{3}\right\|\right)$. Now consider the set $B=\left\{\alpha_{1}, \alpha_{2}, \alpha_{3}\right\}$. It is obvious that $M \in \bigcup\left\{\left\|\neg \alpha_{i}\right\|: \alpha_{i} \in B \cap \mathbf{K}\right\}$. However, on the other hand, $M \notin \bigcup \mathbb{W}_{\mathbf{K} \perp B}$. Indeed assume by redutio that there is some $\mathcal{W} \in \mathbb{W}_{\mathbf{K} \perp B}$ such that $M \in \mathcal{W}$. Then, according to Definition 3.1, there is some $\alpha_{j} \in B \cap \mathbf{K}$ such that $\mathcal{W} \cap\left\|\neg \alpha_{j}\right\|=\{M\}$. Therefore, since $B \cap \mathbf{K}=B=\left\{\alpha_{1}, \alpha_{2}, \alpha_{3}\right\}$ and $M \notin\left\|\neg \alpha_{2}\right\| \cup\left\|\neg \alpha_{3}\right\|$, it must be the case that $\mathcal{W} \cap\left\|\neg \alpha_{1}\right\|=\{M\}$. But, also from Definition 3.1 it follows that there must be some world $N \in \mathcal{W} \cap\left\|\neg \alpha_{2}\right\|$ and then, because $\left\|\neg \alpha_{2}\right\| \subset\left\|\neg \alpha_{1}\right\|$, we conclude that $N \in \mathcal{W} \cap\left\|\neg \alpha_{1}\right\|$ which contradicts $\mathcal{W} \cap\left\|\neg \alpha_{1}\right\|=\{M\}$ (since from $N \in\left\|\neg \alpha_{2}\right\|$ and $M \notin\left\|\neg \alpha_{2}\right\|$ it follows immediately that $N \neq M$ ). Hence $\bigcup\left\{\left\|\neg \alpha_{i}\right\|: \alpha_{i} \in B \cap \mathbf{K}\right\} \nsubseteq \bigcup \mathbb{W}_{\mathbf{K} \perp B}$.

3. Follows trivially from the counterexample presented above.

Proof of Observation 3.9 If $B \cap C n(\emptyset) \neq \emptyset$ or $B \cap \mathbf{K}=\emptyset$ then using Observation 3.2-1. and 2. it can be trivially shown that $\bigcup \mathbb{W}_{\mathbf{K} \perp B}=\bigcup\left\{\left\|\neg \alpha_{i}\right\|: \alpha_{i} \in\right.$ $\left.B_{N} \cap \mathbf{K}\right\}=\emptyset$. Now we assume that $B \cap \operatorname{Cn}(\emptyset)=\emptyset$ and $B \cap \mathbf{K} \neq \emptyset$, and we prove the equality by double inclusion:

(ㄷ) Let $M \in \bigcup \mathbb{W}_{\mathbf{K} \perp B}$, then there is some $\mathcal{W} \in \mathbb{W}_{\mathbf{K} \perp B}$ such that $M \in \mathcal{W}$. Then, according to Definition 3.1 it holds that: (i) $\mathcal{W} \cap\left\|\neg \alpha_{i}\right\| \neq \emptyset$, for all $\alpha_{i} \in B \cap \mathbf{K}$ and (ii) there is some $\alpha_{j} \in B \cap \mathbf{K}$ such that $\mathcal{W} \cap\left\|\neg \alpha_{j}\right\|=\{M\}$. If $\alpha_{j} \in B_{N}$. Then, $\alpha_{j} \in B_{N} \cap \mathbf{K}$ and $M \in\left\|\neg \alpha_{j}\right\|$. Therefore we can conclude that $M \in \bigcup\left\{\left\|\neg \alpha_{i}\right\|: \alpha_{i} \in B_{N} \cap \mathbf{K}\right\}$, and we are done.

If $\alpha_{j} \notin B_{N}$. Then, it follows from Lemma 4.3 that there is some $\alpha_{k} \in$ $B_{N}$ such that $\left\|\neg \alpha_{k}\right\| \subset\left\|\neg \alpha_{j}\right\|$. So, from (i) and (ii) we can conclude that $M \in\left\|\neg \alpha_{k}\right\|$. Furthermore, from $\alpha_{j} \in \mathbf{K}$ and $\left\|\neg \alpha_{k}\right\| \subset\left\|\neg \alpha_{j}\right\|$ we can conclude that $\alpha_{k} \in \mathbf{K}$ (since from $\left\|\neg \alpha_{j}\right\| \cap\|\mathbf{K}\|=\emptyset$ and $\left\|\neg \alpha_{k}\right\| \subset\left\|\neg \alpha_{j}\right\|$ it follows that $\left.\left\|\neg \alpha_{k}\right\| \cap\|\mathbf{K}\|=\emptyset\right)$. Hence, $M \in \bigcup\left\{\left\|\neg \alpha_{i}\right\|: \alpha_{i} \in B_{N} \cap \mathbf{K}\right\}$, as required.

() Follows trivially from Lemma 4.5.

Proof of Observation 3.11 The equality of sets $\|\mathbf{K}\| \cup\left(\cup \mathbb{W}_{\mathbf{K} \perp B}\right)=\|\mathbf{K}\| \cup$ $\left(\bigcup\left\{\left\|\neg \alpha_{i}\right\|: \alpha_{i} \in B_{N} \cap \mathbf{K}\right\}\right)$ follows immediately from Observation 3.9. 
On the other hand, according to Observation 3.10, it holds that $\mathbf{K} \dot{\sim} B=$ $\bigcap\left(\|\mathbf{K}\| \cup\left(\bigcup\left\{\left\|\neg \alpha_{i}\right\|: \alpha_{i} \in B_{N} \cap \mathbf{K}\right\}\right)\right)$. Therefore it follows that $\|\mathbf{K} \dot{\sim} B\| \supseteq$ $\|\mathbf{K}\| \cup\left(\bigcup\left\{\left\|\neg \alpha_{i}\right\|: \alpha_{i} \in B_{N} \cap \mathbf{K}\right\}\right)$.

Now we prove the converse inclusion. If $B \cap \mathbf{K}=\emptyset$, then $\|\mathbf{K} \dot{\sim} B\|=\|\mathbf{K}\| \subseteq$ $\|\mathbf{K}\| \cup\left(\bigcup\left\{\left\|\neg \alpha_{i}\right\|: \alpha_{i} \in B_{N} \cap \mathbf{K}\right\}\right)$ and we are done.

So, let $B \cap \mathbf{K} \neq \emptyset$. Then, making use of Lemma 4.3, it follows trivially that $B_{N} \cap \mathbf{K} \neq \emptyset$. Now let $M \in\|\mathbf{K} \dot{\sim} B\|$ and assume by redutio that $M \notin\|\mathbf{K}\| \cup$ $\left(\bigcup\left\{\left\|\neg \alpha_{i}\right\|: \alpha_{i} \in B_{N} \cap \mathbf{K}\right\}\right)$. Then there is some $\delta \in \mathbf{K}$ such that $\neg \delta \wedge \&\left(B_{N} \cap\right.$ $\mathbf{K}) \in M$ and therefore, because $M$ is consistent, $\delta \vee \neg \&\left(B_{N} \cap \mathbf{K}\right) \notin M$.

On the other hand, since $\delta \vee \neg \&\left(B_{N} \cap \mathbf{K}\right) \in \bigcap\left(\|\mathbf{K}\| \cup\left(\bigcup\left\{\left\|\neg \alpha_{i}\right\|: \alpha_{i} \in\right.\right.\right.$ $\left.\left.\left.B_{N} \cap \mathbf{K}\right\}\right)\right)=\mathbf{K} \dot{\sim} B$, it follows that $M \notin\|\mathbf{K} \dot{\sim} B\|$, which contradicts our hypothesis, and this finishes the proof.

Proof of Observation 3.12 Let $\dot{\sim}$ the operator of full meet multiple contraction on $\mathbf{K}$. Then $\mathbf{K} \dot{\sim} B \subseteq \mathbf{K} \div B$. Hence, by Proposition 2.2-(iii), we can conclude that $\|\mathbf{K} \div B\| \subseteq\|\mathbf{K} \dot{\sim} B\|$. Finally, since $\|\mathbf{K} \dot{\sim} B\|=\|\mathbf{K}\| \cup$ $\left(\bigcup\left\{\left\|\neg \alpha_{i}\right\|: \alpha_{i} \in B_{N} \cap \mathbf{K}\right\}\right)$ (according to Observation 3.11), we can conclude that $\|\mathbf{K} \div B\| \subseteq\|\mathbf{K}\| \cup\left(\bigcup\left\{\left\|\neg \alpha_{i}\right\|: \alpha_{i} \in B_{N} \cap \mathbf{K}\right\}\right)$, as we wished to show.

\section{Proof of Observation 3.13}

(c) Let $\mathcal{W} \in \mathbb{W}_{\mathbf{K} \perp B}$. We must show that $\mathcal{W} \in \mathbb{W}_{\mathbf{K} \perp\left(B_{N} \cap \mathbf{K}\right)}$. Hence, according to Definition 3.1, we must prove that:

(i) $\mathcal{W} \cap\left\|\neg \alpha_{i}\right\| \neq \emptyset$, for all $\alpha_{i} \in B_{N} \cap \mathbf{K}$.

(ii) If $M \in \mathcal{W}$ then there is some $\alpha_{j} \in B_{N} \cap \mathbf{K}$ such that $\mathcal{W} \cap$ $\left\|\neg \alpha_{j}\right\|=\{M\}$.

Condition (i) follows immediately from $\mathcal{W} \in \mathbb{W}_{\mathbf{K} \perp B}$ and Definition 3.1. Now let $M$ be an arbitrary element of $\mathcal{W}$. To show that (ii) holds it we must prove that there is some $\alpha_{j} \in B_{N} \cap \mathbf{K}$ such that $\mathcal{W} \cap\left\|\neg \alpha_{j}\right\|=\{M\}$. Since $\mathcal{W} \in \mathbb{W}_{\mathbf{K} \perp B}$, we have that there is some $\alpha_{i} \in B \cap \mathbf{K}$ such that $\mathcal{W} \cap\left\|\neg \alpha_{i}\right\|=\{M\}$. If $\alpha_{i} \in B_{N}$ we are done. Now we assume that $\alpha_{i} \notin B_{N}$. Then, it follows from Lemma 4.3 that there is some $\alpha_{k} \in B_{N}$ such that $\left\|\neg \alpha_{k}\right\| \subset\left\|\neg \alpha_{i}\right\|$. Hence, since $\mathcal{W} \cap\left\|\neg \alpha_{k}\right\| \neq \emptyset$ (according to Definition 3.1), from $\mathcal{W} \cap\left\|\neg \alpha_{i}\right\|=$ $\{M\}$ we can conclude that $\mathcal{W} \cap\left\|\neg \alpha_{k}\right\|=\{M\}$.

() Let $\mathcal{W} \in \mathbb{W}_{\mathbf{K} \perp\left(B_{N} \cap \mathbf{K}\right)}$. Then conditions (i) and (ii) above are satisfied. Next, in order to show that $\mathcal{W} \in \mathbb{W}_{\mathbf{K} \perp B}$, we prove that:

a. $\mathcal{W} \cap\left\|\neg \alpha_{i}\right\| \neq \emptyset$, for all $\alpha_{i} \in B \cap \mathbf{K}$.

b. If $M \in \mathcal{W}$ then there is some $\alpha_{j} \in B \cap \mathbf{K}$ such that $\mathcal{W} \cap$ $\left\|\neg \alpha_{j}\right\|=\{M\}$.

Condition $a$. follows immediately from (i) and Lemma 4.3. On the other hand condition $b$. is an obvious consequence of (ii) and the fact that $B_{N} \cap \mathbf{K} \subseteq B \cap \mathbf{K}$. 
(2.) Left-to-right: Let $\mathbf{K} \perp B=\mathbf{K} \perp C$. We must show that $\mathbb{W}_{\mathbf{K} \perp B}=\mathbb{W}_{\mathbf{K} \perp C \text {. }}$ But since, according to part (1), this last equality is equivalent to $\mathbb{W}_{\mathbf{K} \perp\left(B_{N} \cap \mathbf{K}\right)}=\mathbb{W}_{\mathbf{K} \perp\left(C_{N} \cap \mathbf{K}\right)}$, it is enough to show the latter of these identities.

Since $\mathbf{K} \perp B=\mathbf{K} \perp C$, it holds that every subset $X$ of $\mathbf{K}$ implies some element of $B$ if and only if $X$ implies some element of $C$ (according to [15, Observation 1.39]). Therefore, it follows from Lemma 4.6 and Definition 3.1 that $\mathbb{W}_{\mathbf{K} \perp\left(B_{N} \cap \mathbf{K}\right)}=\mathbb{W}_{\mathbf{K} \perp\left(C_{N} \cap \mathbf{K}\right)}$, and we are done.

Right-to-left: Let $\mathbb{W}_{\mathbf{K} \perp B}=\mathbb{W}_{\mathbf{K} \perp C}$. It follows immediately from Corollary 3.5 that $\mathbf{K} \perp B=\mathbf{K} \perp C$.

\section{Proof of Theorem 3.14}

Right-to-left $\quad$ Let $\mathbf{K} \div B=T h\left(\|\mathbf{K}\| \cup\left(\bigcup f\left(\mathbb{W}_{\mathbf{K} \perp B}\right)\right)\right)$, for all sets $B$. In order to show that $\div$ is a partial meet multiple contraction we must show that there is some package selection function $\gamma$ for $\mathbf{K}$, such that $\mathbf{K} \div B=\bigcap \gamma(\mathbf{K} \perp B)$. Now let $\gamma$ be the operator defined by $\gamma(\mathbf{K} \perp B)=\{\mathbf{K}\}$ if $\mathbf{K} \perp B=\emptyset$, and $\gamma(\mathbf{K} \perp B)=$ $\left\{T h(\|\mathbf{K}\| \cup \mathcal{W}): \mathcal{W} \in f\left(\mathbb{W}_{\mathbf{K} \perp B}\right)\right\}$ if $\mathbf{K} \perp B \neq \emptyset$. We will show that (a) $\gamma$ is a package selection function for $\mathbf{K}$ and (b) $\mathbf{K} \div B=\bigcap \gamma(\mathbf{K} \perp B)$.

In order to prove (a) we must prove first that $\gamma$ is a function; i.e., if $\mathbf{K} \perp C=$ $\mathbf{K} \perp B$ then $\gamma(\mathbf{K} \perp C)=\gamma(\mathbf{K} \perp B)$ which follows immediately from Observation 3.13-2 and the definition of $\gamma$. Now we must prove that $\gamma$ is a package selection function for $\mathbf{K}$. If $\mathbf{K} \perp B=\emptyset$ then by the definition of $\gamma, \gamma(\mathbf{K} \perp B)=\{\mathbf{K}\}$. Let $\mathbf{K} \perp B \neq \emptyset$. Then $B \cap C n(\emptyset)=\emptyset$ and, due to Observation 3.2-1., it follows that $\mathbb{W}_{\mathbf{K} \perp B} \neq \emptyset$. Then, by Definition 3.3, we have that $\emptyset \neq f\left(\mathbb{W}_{\mathbf{K} \perp B}\right) \subseteq \mathbb{W}_{\mathbf{K} \perp B}$. Theorem 3.4-1. yields $T h(\|\mathbf{K}\| \cup \mathcal{W}) \in \mathbf{K} \perp B$, for all $\mathcal{W} \in f\left(\mathbb{W}_{\mathbf{K} \perp B}\right)$. Hence we can conclude that $\gamma(\mathbf{K} \perp B)$ is a non-empty subset of $\mathbf{K} \perp B$.

To prove (b) we have two cases: Case $1, B \cap C n(\emptyset) \neq \emptyset$. Then $\mathbb{W}_{\mathbf{K} \perp B}=\emptyset$ (by Observation 3.2-1.) and it follows from Definition 3.3 that $\mathbf{K} \div B=T h\left(\|\mathbf{K}\| \cup\left(\bigcup f\left(\mathbb{W}_{\mathbf{K} \perp B}\right)\right)\right)=T h(\|\mathbf{K}\| \cup(\bigcup \emptyset))=T h(\|\mathbf{K}\| \cup \emptyset)=$ $\mathbf{K}$ (where this last equality is justified by Proposition 2.2-(i)). On the other hand, it follows from the definition of $\gamma$ that $\bigcap \gamma(\mathbf{K} \perp B)=\bigcap\{\mathbf{K}\}=\mathbf{K}$. Therefore $\mathbf{K} \div B=\bigcap \gamma(\mathbf{K} \perp B)$.

Case $2, B \cap C n(\emptyset)=\emptyset$. Then $\mathbf{K} \div B=\bigcap\left\{\operatorname{Th}(\|\mathbf{K}\| \cup \mathcal{W}): \mathcal{W} \in f\left(\mathbb{W}_{\mathbf{K} \perp B}\right)\right\}$ and $\gamma(\mathbf{K} \perp B)=\left\{T h(\|\mathbf{K}\| \cup \mathcal{W}): \mathcal{W} \in f\left(\mathbb{W}_{\mathbf{K} \perp B}\right)\right\}$ from which it follows trivially that $\mathbf{K} \div B=\bigcap \gamma(\mathbf{K} \perp B)$. Hence $\div$ is a partial meet multiple contraction on $\mathbf{K}$.

Left-to-right Let $\div$ be a partial meet multiple contraction on $\mathbf{K}$. Then there is some package selection function $\gamma$ for $\mathbf{K}$, such that $\mathbf{K} \div B=\bigcap \gamma(\mathbf{K} \perp B)$, where if $B \cap C n(\emptyset) \neq \emptyset$ then $\gamma(\mathbf{K} \perp B)=\{\mathbf{K}\}$, and if $B \cap C n(\emptyset)=\emptyset$ then $\emptyset \neq$ $\gamma(\mathbf{K} \perp B) \subseteq \mathbf{K} \perp B$. We must prove that there exists a propositional package selection function $f$ for $\|\mathbf{K}\|$ such that $\mathbf{K} \div B=\operatorname{Th}\left(\|\mathbf{K}\| \cup\left(\bigcup f\left(\mathbb{W}_{\mathbf{K} \perp B}\right)\right)\right)$. Let $f$ be defined in the following way: $f\left(\mathbb{W}_{\mathbf{K} \perp B}\right)=\emptyset$ if $B \cap C n(\emptyset) \neq \emptyset$ and $f\left(\mathbb{W}_{\mathbf{K} \perp B}\right)=\left\{\mathcal{W}_{X} \in \mathbb{W}_{\mathbf{K} \perp B}: X=\operatorname{Th}\left(\|\mathbf{K}\| \cup \mathcal{W}_{X}\right) \in \gamma(\mathbf{K} \perp B)\right\}$ if $B \cap \operatorname{Cn}(\emptyset)=$ 
$\emptyset$. We will show that $f$ is a propositional package selection function for $\|\mathbf{K}\|$. Since when $\mathbb{W}_{\mathbf{K} \perp B}=\mathbb{W}_{\mathbf{K} \perp C}$, it follows from Observation 3.13-2 and the definition of $f$ that $f\left(\mathbb{W}_{\mathbf{K} \perp B}\right)=f\left(\mathbb{W}_{\mathbf{K} \perp C}\right)$, we can conclude that $f$ is a function. Now we must prove that $f$ satisfies conditions (1) and (2) of Definition 3.3. That $f\left(\mathbb{W}_{\mathbf{K} \perp B}\right) \subseteq \mathbb{W}_{\mathbf{K} \perp B}$ follows immediately from the definition of $f$. On the other hand, if $\mathbb{W}_{\mathbf{K} \perp B} \neq \emptyset$ it follows from Observation 3.2-1. that $B \cap \operatorname{Cn}(\emptyset)=\emptyset$ and, consequently, $\gamma(\mathbf{K} \perp B) \neq \emptyset$. Then, by Theorem 3.4-2. we can conclude that $\emptyset \neq\left\{\mathcal{W}_{X} \in \mathbb{W}_{\mathbf{K} \perp B}: X=T h\left(\|\mathbf{K}\| \cup \mathcal{W}_{X}\right) \in \gamma(\mathbf{K} \perp B)\right\}=f\left(\mathbb{W}_{\mathbf{K} \perp B}\right)$. Therefore $f$ is a propositional package selection function for $\|\mathbf{K}\|$.

It only remains to show that $\bigcap \gamma(\mathbf{K} \perp B)=T h\left(\|\mathbf{K}\| \cup\left(\bigcup f\left(\mathbb{W}_{\mathbf{K} \perp B}\right)\right)\right)$.

Case $1, B \cap C n(\emptyset) \neq \emptyset$. Then, on the one hand $\gamma(\mathbf{K} \perp B)=\{\mathbf{K}\}$ and, on the other hand, according to Observation 3.2-1., $\mathbb{W}_{\mathbf{K} \perp B}=\emptyset$. Therefore it follows trivially that $\bigcap \gamma(\mathbf{K} \perp B)=T h\left(\|\mathbf{K}\| \cup\left(\bigcup f\left(\mathbb{W}_{\mathbf{K} \perp B}\right)\right)\right)$.

Case 2, $B \cap C n(\emptyset)=\emptyset$. Then it follows from Theorem 3.4-2. and from the definition of $f$ that $\{X: X \in \gamma(\mathbf{K} \perp B)\}=\left\{T h\left(\|\mathbf{K}\| \cup \mathcal{W}_{X}\right): \mathcal{W}_{X} \in\right.$ $\left.f\left(\mathbb{W}_{\mathbf{K} \perp B}\right)\right\}$. Therefore, we obtain that $\bigcap \gamma(\mathbf{K} \perp B)=\bigcap\{X: X \in \gamma(\mathbf{K} \perp B)\}=$ $\bigcap\left\{T h\left(\|\mathbf{K}\| \cup \mathcal{W}_{X}\right): \mathcal{W}_{X} \in f\left(\mathbb{W}_{\mathbf{K} \perp B}\right)\right\}=\bigcap\left\{\bigcap\left(\|\mathbf{K}\| \cup \mathcal{W}_{X}\right): \mathcal{W}_{X} \in f\left(\mathbb{W}_{\mathbf{K} \perp B}\right)\right\}=$ $\bigcap\left(\|\mathbf{K}\| \cup\left(\bigcup f\left(\mathbb{W}_{\mathbf{K} \perp B}\right)\right)\right)=T h\left(\|\mathbf{K}\| \cup\left(\bigcup f\left(\mathbb{W}_{\mathbf{K} \perp B}\right)\right)\right)$, and this finishes the proof.

\section{References}

1. Alchourrón, C., Gärdenfors, P., \& Makinson, D. (1985). On the logic of theory change: Partial meet contraction and revision functions. Journal of Symbolic Logic, 50, 510-530.

2. Alchourrón, C., \& Makinson, D. (1981). Hierarchies of regulations and their logic. In R. Hilpinen (Ed.), New studies in deontic logic: Norms, actions, and the foundations of ethics (pp. 125-148). D. Reidel Publishing Company.

3. Alchourrón, C., \& Makinson, D. (1982). On the logic of theory change: Contraction functions and their associated revision functions. Theoria, 48, 14-37.

4. Chellas, B. F. (1980). Modal logic: An introduction. Cambridge University Press.

5. Fermé, E. \& Reis, M. D. L. System of spheres-based multiple contractions. Journal of Philosophical Logic (this issue).

6. Fuhrmann, A. (1988). Relevant logics, modal logics and theory change. PhD thesis, Australian National University, Camberra.

7. Fuhrmann, A. (1991). Theory contraction through base contraction. Journal of Philosophical Logic, 20, 175-203.

8. Fuhrmann, A., \& Hansson, S. O. (1994). A survey of multiple contraction. Journal of Logic, Language and Information, 3, 39-74.

9. Gärdenfors, P. (1982). Rules for rational changes of belief. In T. Pauli (Ed.), Philosophical essays dedicated to Lennart Áqvist on his fiftieth birthday (No. 34, pp. 88-101). Philosophical Studies.

10. Gärdenfors, P. (1988). Knowledge in flux: Modeling the dynamics of epistemic states. Cambridge: The MIT Press.

11. Gärdenfors, P., \& Makinson, D. (1988). Revisions of knowledge systems using epistemic entrenchment. In M. Y. Vardi (Ed.), Proceedings of the second conference on theoretical aspects of reasoning about knowledge (pp. 83-95). Los Altos: Morgan Kaufmann.

12. Grove, A. (1988). Two modellings for theory change. Journal of Philosophical Logic, 17, $157-170$. 
13. Hansson, S. O. (1989). New operators for theory change. Theoria, 55, 114-132.

14. Hansson, S. O. (1991). Belief base dynamics. PhD thesis, Uppsala University.

15. Hansson, S. O. (1999). A Textbook of belief dynamics. Theory change and database updating. Applied logic series (Vol. 11). Dordrecht: Kluwer Academic Publishers.

16. Niederée, R. (1991). Multiple contraction: A further case against Gärdenfors' principle of recovery. In A. Fuhrmann \& M. Morreau (Eds.), The Logic of theory change (pp. 322-334). Berlin: Springer-Verlag. 